\title{
FINITE APPROXIMATION OF WEYL SYSTEMS
}

\author{
T. DIGERNES, E. HUSSTAD and V. S. VARADARAJAN
}

\begin{abstract}
The functional analytic notion of approximation of Weyl systems, as introduced by Digernes and Varadarajan, is considered. It is shown that the Weyl system on any second countable locally compact abelian group can be approximated by suitably chosen finite Weyl systems (Weyl systems on finite abelian groups).
\end{abstract}

\section{Introduction}

There has in recent years been considerable interest in quantum theories that are analogous to the conventional one, but differ from it in some of their main features. We mention, without aiming at completeness, the following works: Finkelstein [5], and Chan, Finkelstein [2] on $q$-deformed quantum theories; Vladimirov [14], and Vladimirov, Volovich, Zelenov [15] on $p$-adic quantum mechanics; Varadarajan [12] on quantum kinematics over general locally compact abelian groups treated from the point of view of deformation and approximation. Quantum kinematics over finite abelian groups go back to Weyl [18], and Schwinger [9].

In this paper, we develop the point of view in [12] further, as we discuss approximations of quantum kinematics on locally compact abelian groups in more detail.

Our motivation for studying quantum models based on very general abelian groups does not arise solely, or even mainly, from any desire of generality. Rather it stems from the work of Schwinger [9] on the classification of finite quantum systems, and its variations treated in Husstad [4], strongly influenced by Digernes and Varadarajan, and [12]. In [9] and [4], as well as in [18], unitary representations of a finite abelian group $G$ and its dual $\hat{G}$ satisfying Weyl commutation rules (= Weyl system) were studied, and it was shown that the conventional Weyl system associated to $\mathrm{R}^{e}$ may be approximated (as in Section 2) arbitrarily well by Weyl systems on finite abelian groups. The approximation scheme of Schwinger gave remarkable numerical results on the level of generators. Motivated by this, the validity of this ap-

Received December 23, 1996. 
proximation process was proved theoretically by Digernes, Varadarajan, Varadhan [3]. Consequently one could take the point of view that quantum theory associated with a finite abelian group is of much interest, and that the calculations over $\mathrm{R}^{e}$ are idealizations of the finite situation.

Our main Theorem 6.1 states that the Weyl system on any locally compact second countable abelian group is a limit of Weyl systems on finite abelian groups. This motivates dynamical considerations over other groups than $\mathrm{R}^{e}$, as they in this sense also give idealizations of finite quantum systems. Moreover, this scheme makes it possible to obtain numerical results for more unconventional quantum dynamical systems.

It turns out (cfr. the comments in [12]) that the Weyl system on $\mathrm{Z} / p^{n} \mathbf{Z}$ converges to that associated to the $p$-adic field $\mathrm{Q}_{p}$, as $n \longrightarrow \infty$. From this point of view one can for instance study "harmonic oscillators", and "coulomb" problems over local fields and rings. A path-integral formulation for vector spaces over division algebras over non-archimedean local fields has been established in Varadarajan [13].

The paper is organized as follows: In Section 2, Weyl systems and limits of such are defined. "Continuity" results for duality and direct sum are presented, and the structure of finite Weyl systems is discussed.

In Section 3 we approximate any second countable locally compact abelian group $G$ by elementary groups $H_{N} / K_{n} \simeq \mathrm{R}^{e} \oplus \mathrm{T}^{a_{n}} \oplus \mathrm{F}_{N}^{n} \oplus \mathbf{Z}^{b_{N}}, H_{N}$ open compactly generated subgroup, while $K_{n}$ is a compact subgroup for which $G / K_{n}$ is the dual of a compactly generated group. The group $\mathrm{F}_{N}^{n}$ is finite abelian. This follows from the more general results of van Kampen [11], and Pontrjagin [7]. Let $N<n$. Here, $H_{N} \subset H_{n}$ which by [7] in particular induces an injection $\iota_{N}^{n}: Z^{b_{N}} \longrightarrow Z^{b_{n}}$, whereas the natural map $G / K_{N} \longleftarrow G / K_{n}$ induces an injection $\widehat{\kappa_{N}^{n}}: Z^{a_{N}} \longrightarrow Z^{a_{n}}$. In the resulting mixed inductive/projective limit description of $G$, induced maps (for which $\iota_{N}^{n}$, and $\kappa_{N}^{n}$ are two of the matrix coefficients) can be taken to be semi-aligned, but not diagonal in general.

Section 4 is used to define finite abelian groups $G_{n}$ (and maps), candidates for approximating the Weyl system on $G$. We do a two-step approximation in the sense that we first take the diagonal $H_{n} / K_{n}$, and then construct $G_{n}$ based on $H_{n} / K_{n}$ and the matrix coefficients $\iota_{i}^{n}$ and $\widehat{\kappa_{i}^{n}}$, for all $i<n$. We choose to treat circle parts essentially as integer parts through Fourier transforms. In effect, the embedded finite translation on $l^{2}\left(Z^{a_{n}}\right)$, and the embedded finite multiplication by character on $L^{2}\left(T^{a_{n}}\right)$, are intertwined by the non-finite Fourier transform $\mathscr{F}^{n}: l^{2}\left(\mathrm{Z}^{a_{n}}\right) \rightarrow L^{2}\left(\mathrm{~T}^{a_{n}}\right)$.

The space of Schwartz-Bruhat [1] functions $\mathscr{S}(G)$, functions which live on the elementary group $H_{n} / K_{n}$ for some $n$, is introduced in Section 5 to deal with the analysis. The key point is that $\mathscr{S}(G)$ is invariant under the standard 
Weyl system. This moves our calculations to $H_{n} / K_{n}$, and immediately shows that the Weyl system on $G$ can be approximated by Weyl systems on elementary groups. In this section, we get around problems with semi-alignment: Given simple tensors in $\mathscr{S}(N)$ for which we control the support of their images in the discrete space $l^{2}\left(\mathbf{Z}^{a_{N}} \oplus \mathrm{F}_{N}^{N} \oplus \mathbf{Z}^{b_{N}}\right)$, we find that we also control the support of their images in $l^{2}\left(\mathbf{Z}^{a_{n}} \oplus \mathrm{F}_{n}^{n} \oplus \mathbf{Z}^{b_{n}}\right)$ for $n>N$ (Lemma 5.3). Their support is governed by the maps $\iota_{N}^{n}$ and $\widehat{\kappa_{N}^{n}}$.

In Section 6, the general approximation result is proved. First, pointwise convergence of characters is verified. The important point is that in Z-directions, the approximation is exact from some $n$, and by semi-alignment we control the coordinate in these directions. In T-directions we have no such control, but the result follows as the approximation in T-directions is uniform. The strong convergence of projections follows directly from the support control of Section 5. The remaining statements essentially follow from pointwise convergence of characters, and the support control Lemma 5.3. For the sake of completeness, we conclude with a proof for the conventional case $\mathbf{R}^{e}$. Finally, some applications to local fields and rings are mentioned.

\section{Limits of Weyl Systems}

Let $G$ be a second countable locally compact abelian group, with $\hat{G}$ as its Pontrjagin dual. The Weyl representations $V$ and $U$ of $G$ and $\hat{G}$, respectively, are, for $x \in G$ and $\gamma \in \hat{G}$, given by

$$
\begin{aligned}
& (V(x) f)(y)=f(y-x), \\
& (U(\gamma) f)(y)=\langle y, \gamma\rangle f(y), \quad f \in L^{2}(G), \quad y \in G .
\end{aligned}
$$

This pair of strongly continuous unitary representations satisfies the Weyl relations;

$$
U(\gamma) V(x)=\langle x, \gamma\rangle V(x) U(\gamma) \quad x \in G, \quad \gamma \in \hat{G} .
$$

The pair $(V, U)$ is called the standard Weyl system on $G$. The standard Weyl system is irreducible; the resulting projective unitary representation of $G \oplus \hat{G}$ has no non-trivial invariant subspaces in $L^{2}(G)$.

Definition 2.1 (Limit of Weyl systems). Let $\left\{G_{n}\right\}_{n=1}^{\infty}, G$ be second countable, locally compact abelian groups with associated standard Weyl systems $\left\{\left(V_{n}, U_{n}\right)\right\}$ and $(V, U)$. Then we say that the sequence $\left\{G_{n}\right\}$ converges to $G$ in the sense of Weyl systems (or that $(V, U)$ on $G$ is the limit of $\left(V_{n}, U_{n}\right)$ on $\left.G_{n}\right)$ if the following conditions are satisfied:

i) There is a Hilbert space $\mathfrak{H}$ and isometries $I_{n}: L^{2}\left(G_{n}\right) \longrightarrow \mathfrak{H}$, 
$I: L^{2}(G) \longrightarrow \mathfrak{H}$, such that $P_{n} \longrightarrow P$ strongly. Here, $P_{n}$ and $P$ are the orthogonal projections on $I_{n}\left(L^{2}\left(G_{n}\right)\right)$ and $I\left(L^{2}(G)\right)$, respectively.

ii) Setting

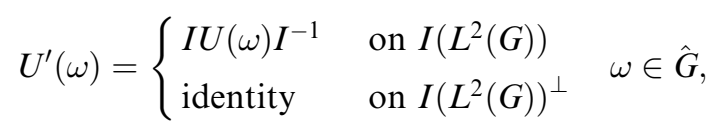

and defining $V^{\prime}, U_{n}^{\prime}$ and $V_{n}^{\prime}$ similarly, there are, for each $x \in G, \gamma \in \hat{G}$, sequences $\left\{x^{n}\right\}$ and $\left\{\gamma^{n}\right\}$ such that $x^{n} \in G_{n}, \gamma^{n} \in \hat{G}_{n}$, and

$$
U_{n}^{\prime}\left(\gamma^{n}\right) \longrightarrow U^{\prime}(\gamma), \quad V_{n}^{\prime}\left(x^{n}\right) \longrightarrow V^{\prime}(x)
$$

strongly.

If the conditions in Definition 2.1 are satisfied, we easily see that $\left\langle x^{n}, \gamma^{n}\right\rangle \longrightarrow\langle x, \gamma\rangle$, so pointwise convergence of characters is necessary for Weyl convergence.

Assume that the standard Weyl system on $G$ is a limit of the standard Weyl systems on $G_{n}$. The Stone-von Neumann-Mackey Theorem says that up to multiplicity and unitary equivalence, the Weyl relations for $G$ have a unique solution. Thus, in the natural sense we can approximate any Weyl system on $G$ (= any other solution of (1)) by Weyl systems on $G_{n}$. In this paper, we exclusively work with standard Weyl systems.

2.1. Duality. The standard Weyl system $(\hat{V}, \hat{U})$ on $\hat{G}$ (identify $G$ and its bidual) is connected to the standard Weyl system $(V, U)$ on $G$ through the Fourier transform; $\hat{V}=\mathscr{F} U \mathscr{F}^{-1}$ and $\hat{U}=\mathscr{F} V \mathscr{F}^{-1}$. The Fourier transform $\mathscr{F}: L^{2}(G) \longrightarrow L^{2}(\hat{G})$ is for suitable $f$ given by $(\mathscr{F} f)(\gamma)=\int_{x \in G} f(x)\langle-x, \gamma\rangle d x$, $\gamma \in \hat{G}$.

Proposition 2.2. If $G$ is a limit (Definition 2.1) for $G_{n}$, then $\hat{G}$ is a limit for $\hat{G}_{n}$ in the sense of Weyl systems.

Proof. Define $\hat{I}_{n}: L^{2}\left(\hat{G}_{n}\right) \longrightarrow \mathfrak{H}$ and $\hat{I}: L^{2}(\hat{G}) \longrightarrow \mathfrak{H}$ by $\hat{I}_{n}=I_{n} \mathscr{F}_{n}^{-1}$ and $\hat{I}=I \mathscr{F}^{-1}$. By construction, $\hat{P}_{n}=P_{n}$ and $\hat{P}=P$. Let $x \in G$. Then we easily see that $\hat{I}_{n} \hat{U}_{n}\left(x^{n}\right) \hat{I}_{n}^{-1}=I_{n} V_{n}\left(x^{n}\right) I_{n}^{-1}$ on $P_{n}(\mathfrak{H})$ and $\hat{I} \hat{U}(x) \hat{I}^{-1}=I V(x) I^{-1}$ on $P(\mathfrak{H})$. Similar formulas for $\hat{V}$ prove the proposition.

2.2. Direct Sum. If $G$ is decomposable, say the direct sum of two subgroups, $G=G_{1} \oplus G_{2}$, then the standard Weyl system of $G$ can be identified with the sum of the standard Weyl systems $\left(V_{G_{j}}, U_{G_{j}}\right)$ on $G_{j}, j=1,2$. This means, for $x=\left(x_{1}, x_{2}\right) \in G, \quad L^{2}(G) \simeq L^{2}\left(G_{1}\right) \otimes L^{2}\left(G_{2}\right)$ and $V_{G}(x) \simeq$ $V_{G_{1}}\left(x_{1}\right) \otimes V_{G_{2}}\left(x_{2}\right)$. Similar relations hold for $U$. This extends to finite index sets. 
Proposition 2.3. If $\left(V^{j}, U^{j}\right)$ on $G^{j}$ is the limit of $\left(V_{n}^{j}, U_{n}^{j}\right)$ on $G_{n}^{j}$ for $j$ in a finite set, then $(V, U)$ on $G=\oplus_{j} G^{j}$ is the limit of $\left(V_{n}, U_{n}\right)$ on $G_{n}=\oplus_{j} G_{n}^{j}$.

PRoof. The result follows from strong continuity of tensor products for uniformly bounded sequences of operators. Let us give some details in the case of two summands: Define $I_{n}=I_{n}^{1} \otimes I_{n}^{2}$, and $I=I^{1} \otimes I^{2}$, both acting on $\mathfrak{H}:=\mathfrak{H}^{1} \otimes \mathfrak{H}^{2}$. Then $P_{n}=P_{n}^{1} \otimes P_{n}^{2}$ and $P=P^{1} \otimes P^{2}$. Likewise, for $x=$ $\left(x_{1}, x_{2}\right) \in G$, put $x^{n}=\left(x_{1}^{n}, x_{2}^{n}\right)$. Applied to a simple tensor $\psi=\psi_{1} \otimes \psi_{2} \in \mathfrak{H}$, $V_{n}^{\prime}\left(x^{n}\right) \psi=V_{n}^{\prime}\left(x_{1}^{n}\right) \psi_{1} \otimes V_{n}^{\prime}\left(x_{2}^{n}\right) \psi_{2}$ and $V^{\prime}(x) \psi=V^{\prime}\left(x_{1}\right) \psi_{1} \otimes V^{\prime}\left(x_{2}\right) \psi_{2}$. As $P_{n}^{j}$ and $V_{n}^{\prime}\left(x_{j}^{n}\right)$ are uniformly bounded in norm, we get the expected convergence. The arguments are the same for $U$ when we take $\gamma^{n}=\left(\gamma_{1}^{n}, \gamma_{2}^{n}\right)$ for $\gamma=\left(\gamma_{1}, \gamma_{2}\right) \in \hat{G}=\hat{G}_{1} \oplus \hat{G}_{2}$.

2.3. Finite Weyl systems. Let $n=p_{1}^{r_{1}} p_{2}^{r_{2}} \cdots p_{k}^{r_{k}}$ be the prime expansion of $n$ ( $p_{j}$ are different primes while $r_{j}$ are non-negative integers). Then, $\mathbf{Z}_{n} \simeq \mathbf{Z}_{p_{1}^{r_{1}}} \oplus \mathbf{Z}_{p_{2}^{r_{2}}} \oplus \cdots \oplus \mathbf{Z}_{p_{k}^{r_{k}}}$, and $\mathbf{Z}_{n}$ is indecomposable if and only if $n$ is a prime power.

So, recalling the direct sum construction in the previous paragraph, the standard Weyl system $(V, U)$ on $Z_{n}$ is indecomposable in this geometrical sense, precisely when $n=p^{r}$ is a prime power. By their structure theory, namely as direct sums of finite cyclic groups, we can build the Weyl system on any finite abelian group from these geometrically indecomposable finite Weyl systems. Schwinger [9] started constructing this theory of finite degree of freedom. Finite quantum systems were also studied by Štovíček and Tolar [10], and later in [4].

\section{Structure of Second Countable Locally Compact Abelian Groups}

Recall structure theorems on 1.c.a. groups: Pontrjagin [7], Section 39, Theorem 51 proves that any compactly generated group is of the form $\mathrm{R}^{e} \oplus C \oplus \mathbf{Z}^{b}$, where $C$ is a compact abelian group ("compactly generated" means "generated by a compact neighbourhood of the identity"). In the same reference, Section 39, Proposition A, he proves that for any 1.c.a. group $G$, and any compact set $K \subset G$, there is a compactly generated open subgroup $H$ such that $K \subset H \subset G$. Moreover, the structure theorem of van Kampen [11], Theorem 2, says that any 1.c.a. group $G$ is of the form $\mathrm{R}^{e} \oplus G^{1}$, where $G^{1}$ contains a compact open subgroup $K$. For any other such decomposition, the exponent $e$ is the same. Following Reiter [8], we say that $G$ is a $G^{1}$-group if $e=0$ in this decomposition. In particular, a compactly generated $G^{1}$-group is of the form $C \oplus \mathbf{Z}^{b}$, where $C$ is compact.

If $G$ is second countable and 1.c.a., so is $\hat{G}$ and any subgroup and quotient 
of $G$. Likewise, the property of being a $G^{1}$-group is preserved under these operations.

We have not found Proposition 3.2 in any standard source in topological group theory. That proposition follows from the next lemma, which is probably also stated somewhere.

Lemma 3.1. Let $G^{1}$ be a $G^{1}$-group, $H \subset G^{1}$ a compactly generated open subgroup, and let $K^{\prime}$ be a compactly generated open subgroup of $\widehat{G}^{1}$. If $K:=\left(K^{\prime}\right)^{\perp} \subset H$ (the annihilator is taken in $\left.\widehat{G^{1}}\right)$, then $H / K \simeq \mathrm{T}^{a} \oplus \mathbf{F} \oplus \mathbf{Z}^{b}$, an elementary group ( $\mathrm{F}$ is a finite abelian group).

Proof. Use the structure theorem of Pontrjagin [7], Section 39, Theorem 51, for compactly generated groups on both $H$ and $K^{\prime}$. By duality, $G^{1} / K \simeq \mathrm{T}^{a} \oplus D$, where $D$ is a discrete abelian group. Moreover, as $K$ is compact, $H / K \simeq C / K \oplus \mathbf{Z}^{b}$, where $C / K$ is a compact group. As $H \subset G^{1}$ is open, $H / K \subset G^{1} / K$ is open, and there is an open injection $C / K \oplus \mathbf{Z}^{b} \longrightarrow \mathrm{T}^{a} \oplus D$. In particular, the compact open subgroup $C / K \oplus\{0\}$ must map to a compact open subgroup. As $\mathrm{T}^{a}$ has no open subgroups but itself, the image of $C / K \oplus\{0\}$ is of the form $\mathrm{T}^{a} \oplus F$, where $F \subset D$ is discrete, but also compact. Thus $F$ is finite.

Proposition 3.2. Let $G$ be a second countable locally compact abelian group. Then $G \simeq \mathrm{R}^{e} \oplus G^{1}$, where the following is true for the group $G^{1}$ : There exists an increasing sequence of open subgroups $\left\{H_{n}\right\}_{n=1}^{\infty}$ such that $\cup H_{n}=G^{1}$, and a decreasing sequence of compact subgroups $\left\{K_{n}\right\}, H_{1} \supset K_{n} \supset K_{n+1}$, such that $\cap K_{n}=\{0\}$. Moreover, $H_{n} / K_{n} \simeq \mathrm{T}^{a_{n}} \oplus \mathrm{F}_{n} \oplus \mathbf{Z}^{b_{n}}$, where $\mathrm{F}_{n}$ is a finite abelian group $\left(a_{n}\right.$ and $b_{n}$ are non-negative integers).

Proof. The first part follows from [11].

Any separable 1.c.a. group can be written as a countable union of compact sets (take an open neighbourhood of 0 with compact closure, and translate this closure with elements from a countable dense subset of $G^{1}$ ). Thus, by [7], Section 39, Proposition A, and Theorem 51, there is $\left\{H_{n}^{\prime \prime}\right\}$ such that $\cup H_{n}^{\prime \prime}=G^{1}$, and $H_{n}^{\prime \prime}$ is open and compactly generated. Let $H_{n}^{\prime}=H_{1}^{\prime \prime}+$ $H_{2}^{\prime \prime}+\cdots+H_{n}^{\prime \prime}$, this subgroup is also open and compactly generated. Then $H_{n}^{\prime} \subset H_{n+1}^{\prime}$. Likewise, construct $\left\{K_{m}^{\prime}\right\}$ such that $K_{m}^{\prime}$ is open and compactly generated, and $K_{m}^{\prime} \nearrow \widehat{G^{1}}$. Thus, $K_{m}:=\left(K_{m}^{\prime}\right)^{\perp}$ is a compact subgroup of $G^{1}$ such that $K_{m} \searrow\{0\}$. As $K_{1}$ is compact and $\left\{H_{n}^{\prime}\right\}$ covers $G^{1}$, there is an integer $N$ such that for $n>N, K_{1} \subset H_{n}^{\prime}$. Let $H_{n}:=H_{N+n}^{\prime}$. Then, by the previous lemma, we are done.

The proof of Lemma 3.1 implies that $H_{n} / K_{m} \simeq \mathrm{T}^{a_{m}} \oplus \mathrm{F}_{n}^{m} \oplus \mathbf{Z}^{b_{n}}$, so we get elementary groups also if $n \neq m$. Here, $\mathrm{F}_{n}^{n}=\mathrm{F}_{n}$. 
3.1. Semi-alignment for $G^{1}$-groups. Let $G^{1}$ be a second countable $G^{1}$-group with $\left\{H_{n}\right\}$ and $\left\{K_{m}\right\}$ from Proposition 3.2. Let $\pi_{m}: G^{1} \longrightarrow G^{1} / K_{m}$ and $\pi_{l m}: G^{1} / K_{m} \longrightarrow G^{1} / K_{l}$ be the natural maps $(m>l)$. We have $\pi_{l}=\pi_{m} \circ \pi_{l m}$, and the kernel of $\pi_{l m}$ is the compact group $K_{l} / K_{m}$. From the proof of Lemma 3.1, there are subgroups $Z_{n}, C_{n} \subset G^{1}$, where $Z_{n} \simeq Z^{b_{n}}, C_{n}$ is compact, such that $H_{n}=C_{n}+Z_{n}$ (direct sum). Likewise, there are subgroups $T_{m}, D_{m} \subset G / K_{m}$ with $T_{m} \simeq \mathrm{T}^{a_{m}}, D_{m}$ is discrete, such that $G / K_{m}=T_{m}+D_{m}$ (direct sum). For $m>l$ and $k>n$ we then have the following commuting diagram, which describes the structure of $G^{1}$ in terms of elementary groups; the top row gives $G^{1}$ as an inductive limit, while the right-most column describes $G^{1}$ as a projective limit:

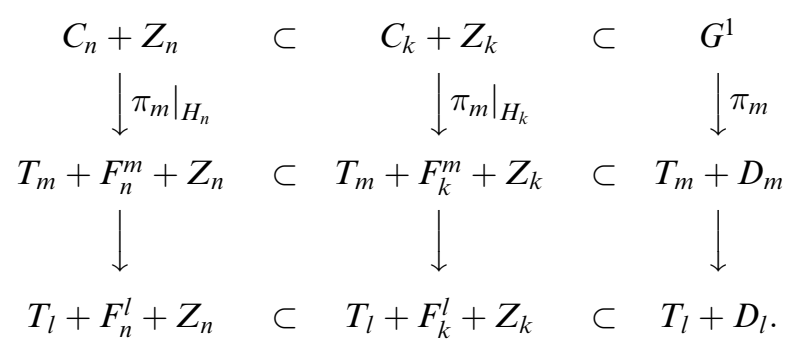

All sums are direct. By the subgroup $Z_{n} \subset G^{1} / K_{m}$ we mean the isomorphic image of $Z_{n}$ under $\pi_{m}$. The subgroups $F_{n}^{l} \simeq \mathrm{F}_{n}^{l}$ are finite. This setup follows from the comment following Proposition 3.2. However, in this diagram we select a basis for all $\mathrm{Z}^{b_{n}}$-parts from that in the top row, and a basis on all $\mathrm{T}^{a_{n}}$ parts from that in the right-most column. We make no particular choice for the finite parts. Thus, in this basis the inclusion $H_{n} / K_{m} \subset H_{k} / K_{m}$ is represented by a $3 \times 3$-matrix

$$
i_{n k}^{m}=\left(\begin{array}{ccc}
\text { id } & & \\
& \theta_{n k}^{m} & \phi_{n k}^{m} \\
& & \iota_{n}^{k}
\end{array}\right),
$$

for some morphisms $\theta_{n k}^{m}: F_{n}^{m} \longrightarrow F_{k}^{m}, \phi_{n k}^{m}: Z_{n} \longrightarrow F_{k}^{m}$, and $\iota_{n}^{k}: Z_{n} \longrightarrow Z_{k}$. The empty places represent 0 -maps. The $2 \times 2$-matrix in the lower right corner is triangular because $Z_{k}$ has no finite subgroups.

Lemma 3.3. In the preceeding matrix representation, $\theta_{n k}^{m}, \iota_{n}^{k}$ are both injective.

Proof. The map $\theta_{n k}^{m}$ is injective as the finite part is only mapped into the finite part.

Let $0 \neq z \in Z_{n}$. Let $[z]$ be the image of $z$ in $Z_{n} / \operatorname{Ker}\left(\phi_{n k}^{m}\right) \simeq \operatorname{Im}\left(\phi_{n k}^{m}\right)$. As this quotient is a finite abelian group, there is some integer $k$ such that 
$[k z]=k[z]=0$. Consequently, $k z \in \operatorname{Ker}\left(\phi_{n k}^{m}\right)$. Thus, by injectivity of $i_{n k}^{m}$, $0 \neq i_{n k}^{m}(0,0, k z)=\left(0,0, \iota_{n}^{k}(k z)\right)$, so $\iota_{n}^{k}(z) \neq 0$. Thus, $\iota_{n}^{k}$ is injective.

Let us recall some generalities on dual groups. For 1.c.a. groups $\left\{G_{i}\right\}(i$ runs over a finite set), set $G=\oplus_{i} G_{i}$. Then $\widehat{G}=\widehat{\oplus}_{i} G_{i} \simeq \oplus_{i} \widehat{G}_{i}$. Duality between $G$ and $\widehat{G}:=\oplus_{i} \widehat{G}_{i}$ is set up with

$$
\left\langle\left(x_{i}\right),\left(\gamma_{j}\right)\right\rangle=\prod_{i}\left\langle x_{i}, \gamma_{i}\right\rangle_{G_{i}}
$$

where for $x_{i} \in G_{i}$ and $\gamma_{i} \in \widehat{G}_{i},\left\langle x_{i}, \gamma_{i}\right\rangle_{G_{i}}$ is some duality between $G_{i}$ and $\widehat{G}_{i}$. Let $\alpha: G \longrightarrow X$ be a morphism between 1.c.a. groups $G=\oplus_{i} G_{i}$ and $X=\oplus_{j} X_{j}$. Thus, $\alpha=\left(\alpha_{i j}\right)$, where $\alpha_{i j}: G_{j} \longrightarrow X_{i}$ is a morphism. Then the dual map $\widehat{\alpha}: \widehat{X} \longrightarrow \widehat{G}$ (under (4)) has matrix representation $\widehat{\alpha}=\left(\widehat{\alpha_{j i}}\right)$ in the natural dual basis on both $\widehat{X}$, and $\widehat{G}$. Here, $\widehat{\alpha_{i j}}: X_{i} \longrightarrow G_{j}$ is the dual map under $\langle\cdot, \cdot\rangle_{G_{j}}$, and $\langle\cdot, \cdot\rangle_{X_{i}}$. Thus, the rule is the same as the usual one for the adjoint in matrix algebras.

So, $\left.\pi_{l m}\right|_{H_{n} / K_{m}}=: j_{l m}^{n}: H_{n} / K_{m} \longrightarrow H_{n} / K_{l}$ has matrix representation

$$
j_{l m}^{n}=\left(\begin{array}{ccc}
\kappa_{l}^{m} & \phi_{l m}^{\prime n} & \\
& \theta_{l m}^{\prime n} & \\
& & \mathrm{id}
\end{array}\right) .
$$

The reason is that the dual of $j_{l m}^{n}$ is an open injection; of the same type as $i_{n k}^{m}$. Using (4):

COROLlary 3.4. In this matrix representation for $j_{l m}^{n}, \kappa_{l}^{m}: T_{m} \longrightarrow T_{l}$ and $\theta_{l m}^{\prime n}: F_{n}^{m} \longrightarrow F_{n}^{l}$ are both (non-zero) surjective. The morphism $\phi_{l m}^{\prime n}: F_{n}^{m} \longrightarrow T_{l}$ could be 0 .

The reason why $\iota_{n}^{k}$, and $\kappa_{l}^{m}$ do not depend on $m$ and $n$, respectively, is that all diagrams in (2) are commutative. In fact, $\iota_{n}^{k}$ represents the inclusion of $Z_{n}$ in $H_{n}$ into $Z_{k}$ in $H_{k}$ (top row). Similarly, $\kappa_{l}^{m}$ is the matrix coefficient in $\pi_{l m}$ mapping $T_{m}$ onto $T_{l}$ (right most column).

Later, we need some additional technical properties on the description of $G^{1}$.

Lemma 3.5. Let $i: Z^{b} \longrightarrow Z^{b^{\prime}}$ be an injection. Then there is a complemented submodule $B$ such that $i\left(\mathrm{Z}^{b}\right) \subset B \subset \mathrm{Z}^{b^{\prime}}, B \simeq \mathrm{Z}^{b}$.

Proof. Let $B$ be those $z \in Z^{b^{\prime}}$ for which $n z \in i\left(Z^{b}\right)$ for some $n \in Z$. This is the torsion closure of the image of $i$. It is easy to see that $B$ is a submodule. Let non-zero $n_{j} u_{j} \in i\left(\mathbf{Z}^{b}\right)$ for $n_{j} \in \mathbf{Z}, u_{j} \in B$, where $j$ runs over a finite set. If $\left\{n_{j} u_{j}\right\}$ is dependent over $Z$, then $\left\{u_{j}\right\}$ is also dependent over Z. Conversely, as 
$i\left(Z^{b}\right) \subset B$, the rank of $B$ over $Z$ equals the rank of $i\left(Z^{b}\right)$ over $Z$. As $i$ is injective, this rank is $b$. Moreover, it follows from the definition of $B$ that the quotient $Z^{b^{\prime}} / B$ is torsion free. This implies that $B$ is a direct summand (with $b$ generators).

LemmA 3.6. Let $Z^{b_{1}} \stackrel{\iota_{1}}{\longrightarrow} Z^{b_{2}} \ldots Z^{b_{n}} \stackrel{\iota_{n}}{\longrightarrow} Z^{b_{n+1}} \stackrel{\iota_{n+1}}{\longrightarrow} \ldots$ where $\left\{\iota_{n}\right\}$ consists of injections. Then we can choose the basis on each $Z^{b_{n}}$ such that for any pair $m>n$, for $\iota_{n}^{m}:=\iota_{m-1} \circ \cdots \circ \iota_{n}, \iota_{n}^{m}\left(Z^{b_{n}}\right) \subset Z^{b_{n}} \oplus\{0\}^{b_{m}-b_{n}} ;$ maps into the first $b_{n}$ factors.

Proof. Choose a basis on $Z^{b_{1}}$. Then use Lemma 3.5 to find complemented $\iota_{1}\left(Z^{b_{1}}\right) \subset B_{1} \subset Z^{b_{2}}$. Now it is clear that we can choose a basis as wanted on $Z^{b_{2}}$. Again from Lemma 3.5 we find complemented $\iota_{2}\left(Z^{b_{2}}\right) \subset B_{2} \subset Z^{b_{3}}$. In particular, $\iota_{2}\left(B_{1}\right) \subset B_{2}$. Use Lemma 3.5 once more to find complemented $\iota_{2}\left(B_{1}\right) \subset B_{2}^{1} \subset B_{2}$. Now we can obviously choose a basis on $Z^{b_{3}}$ where $Z^{b_{2}}$ is mapped into the first $b_{2}$ coordinates, while $Z^{b_{1}}$ is mapped into the first $b_{1}$ coordinates of $Z^{b_{3}}$. The proof is completed by continuing this construction.

Define the elementary groups $E_{n m}:=\mathrm{T}^{a_{m}} \oplus \mathrm{F}_{n}^{m} \oplus \mathrm{Z}^{b_{n}}, \quad \mathrm{~F}_{n}^{n}=\mathrm{F}_{n} \quad$ and $E_{n}:=E_{n n}$. Then $H_{n} / K_{m} \simeq E_{n m}$.

Let us return to Diagram (2). Using this, we can assume that the induced injection (same notation) $i_{n k}^{m}: E_{n m} \longrightarrow E_{k m}$ has a matrix representation like (3), and the induced surjection $j_{l m}^{n}: E_{n m} \longrightarrow E_{n l}$ has a matrix representation like (5).

As $\iota_{n}^{k}: Z^{b_{n}} \longrightarrow Z^{b_{k}}$ is given as in Lemma 3.6, we apply that lemma to $\left\{Z^{b_{n}}\right\}$. We use this change of basis on $Z^{b_{n}}$ in any $E_{n l}$. Thus, the matrix representation of $i_{n k}^{m}$ is of the same type as before. As a dual condition (use (4) between $Z^{a_{n}}$ and $\mathrm{T}^{a_{n}}$ ), we make the kernel of $\kappa_{l}^{m}$ contain $\mathrm{T}^{a_{m}-a_{l}} \oplus\{0\}^{a_{l}}$. The annihilator of the image of a morphism is the kernel of the dual map. The observation that relates all $\mathrm{Z}^{b_{n}}$ (in $H_{n} / K_{l}$ ) to $H_{n}$ is crucial at this point. Ordering problems would otherwise occur for the two-dimensional array (those $(k, l) \in \mathbf{Z}^{2}$ for which $\left.k, l>0\right)$, and we could not get the analogue of Lemma 3.6.

Assume that $n>N$ are positive integers. Rename $i_{N n}:=i_{N n}^{n}$ and $j_{N n}:=j_{N n}^{N}$. The next result summarizes our discussion:

Proposition 3.7. (semi-alignment). Let $G^{1}$ be a $G^{1}$-group with structure given by Proposition 3.2. Then $H_{N} / K_{n} \simeq E_{N n}=\mathrm{T}^{a_{n}} \oplus \mathrm{F}_{N}^{n} \oplus \mathrm{Z}^{b_{N}}$, where for $n>N$ the induced injection $i_{N n}: E_{N n} \longrightarrow E_{n}$, and the induced surjection $j_{N n}: E_{N n} \longrightarrow E_{N}$ can be assumed to have matrix representations 


$$
i_{N n}=\left(\begin{array}{ccc}
\mathrm{id} & & \\
& \theta_{N}^{n} & \phi_{N}^{n} \\
& & \iota_{N}^{n}
\end{array}\right) \quad \text { and } \quad j_{N n}=\left(\begin{array}{ccc}
\kappa_{N}^{n} & \phi_{N}^{\prime n} & \\
& \theta_{N}^{\prime n} & \\
& & \mathrm{id}
\end{array}\right) \text {. }
$$

The maps $\theta_{N}: \mathrm{F}_{N} \longrightarrow \mathrm{F}_{n}$ and $\iota_{N}^{n}: \mathbf{Z}^{b_{N}} \longrightarrow \mathrm{Z}^{b_{n}}$ are injective, and $\iota_{N} n\left(\mathbf{Z}^{b_{N}}\right) \subset$ $\mathbf{Z}^{b_{N}} \oplus\{0\}^{b_{n}-b_{N}}$. Furthermore, $\theta_{N}^{\prime n}: \mathrm{F}_{N}^{n} \longrightarrow \mathrm{F}_{N}$ and $\kappa_{N}^{n}: \mathrm{T}^{a_{n}} \longrightarrow \mathrm{T}^{a_{N}}$ are surjective. Also, the kernel of $\kappa_{N}^{n}$ contains $\mathrm{T}^{a_{n}-a_{N}} \oplus\{0\}^{a_{N}}$. The morphisms $\phi_{N}^{n}: \mathrm{Z}^{b_{n}} \longrightarrow \mathrm{F}_{n}$ and $\phi_{N}^{\prime n}: \mathrm{F}_{N}^{n} \longrightarrow \mathrm{T}^{a_{N}}$ could be 0 .

As $\phi_{N}^{n}$ and $\phi_{N}^{\prime n}$ in general are non-zero, the finite parts may intertwine in a non-trivial way. This causes technical problems in the rest of this paper.

3.2. The dual system. This paragraph contains definitions.

From standard topological group theory, $\mathrm{Z}^{a_{n}} \oplus \mathrm{F}_{N}^{n} \oplus \mathrm{T}^{b_{N}} \simeq \widehat{H_{N} / K_{n}} \simeq$ $K_{n}^{\perp} / H_{N}^{\perp}$. The sequence $\left\{K_{n}^{\perp}\right\} \nearrow \widehat{G^{1}}$ consists of open subgroups while the elements of $\left\{H_{N}^{\perp}\right\} \searrow\{0\}$ are compact subgroups. So, the (non-unique) sequences $\left\{H_{N}\right\}$ and $\left\{K_{n}\right\}$ single out a special decomposition for the dual group $\widehat{G^{1}}$.

Fix a duality $\langle\cdot, \cdot\rangle$ between $G^{1}$ and $\widehat{G^{1}}$. Let $x \in H_{N}$ and $\gamma \in K_{n}^{\perp}$. Then

$$
\left\langle x+K_{n}, \gamma+H_{N}^{\perp}\right\rangle_{N n}=\langle x, \gamma\rangle
$$

(well) defines the duality $\langle\cdot, \cdot\rangle_{N n}$ between $H_{N} / K_{n}$ and $\widehat{H_{N} / K_{n}}=K_{n}^{\perp} / H_{N}^{\perp}$.

Define the standard duality $\langle\cdot, \cdot\rangle_{s}$ between $E_{N n}=\mathrm{T}^{a_{n}} \oplus \mathrm{F}_{N}^{n} \oplus \mathbf{Z}^{b_{N}}$ and $\widehat{E_{N n}}=Z^{a_{n}} \oplus \mathrm{F}_{N}^{n} \oplus \mathrm{T}^{b_{N}} \quad$ (dual basis) as follows: Let $x=(t, f, z)=$ $\left(\left(t_{i}\right),\left(f_{j}\right),\left(z_{k}\right)\right) \in E_{N n}$, and $\gamma=(u, g, s)=\left(\left(u_{i}\right),\left(g_{j}\right),\left(s_{k}\right)\right) \in \widehat{E_{N n}}$. Then

$$
\langle x, \gamma\rangle_{s}=\langle t, u\rangle_{\mathrm{T}^{a_{n}}}\langle f, g\rangle_{\mathrm{F}_{N}^{n}}\langle z, s\rangle_{\mathrm{Z}^{b_{n}}}=\prod_{i} t_{i}^{u_{i}} \prod_{j} e^{2 \pi \frac{f_{i} g_{j}}{n_{j}}} \prod_{k} s_{k}^{z_{k}},
$$

$\mathrm{F}_{N}^{n}=\oplus_{j} Z_{n_{j}}$ from the structure theorem of finite abelian groups.

In the previous section we found an isomorphism $H_{N} / K_{n} \simeq E_{N n}$ giving Proposition 3.7. Let

$$
x+K_{n} \in H_{N} / K_{n} \longrightarrow x_{N n} \in E_{N n}, \quad \text { in particular } \quad x_{n}:=x_{n n}
$$

denote this isomorphism. There is an isomorphism between $K_{n}^{\perp} / H_{N}^{\perp}$ and $\widehat{E_{N n}}$,

$$
\gamma+H_{N}^{\perp} \in K_{n}^{\perp} / H_{N}^{\perp} \longrightarrow \gamma_{N n} \in \widehat{E_{N n}}, \quad \text { in particular } \quad \gamma_{n}:=\gamma_{n n},
$$

such that for $x \in x+K_{n}$, and $\gamma \in \gamma+H_{N}^{\perp}$,

$$
\langle x, \gamma\rangle=\left\langle x+K_{n}, \gamma+H_{N}^{\perp}\right\rangle_{N n}=\left\langle x_{N n}, \gamma_{N n}\right\rangle_{s} .
$$

This is because there is only one dual pairing modulo automorphisms (for any isomorphism $K_{n}^{\perp} / H_{N}^{\perp} \simeq \widehat{E_{N n}}$, (12) defines some dual pairing, compose this isomorphism with the appropriate automorphism). 
Assume $N<n$. Then $\widehat{i_{N n}}$ (under the standard dual pairing) is the surjection induced from the natural map $K_{n}^{\perp} / H_{n}^{\perp} \longrightarrow K_{n}^{\perp} / H_{N}^{\perp}$, and $\widehat{j_{N n}}$ is the injection induced from the inclusion $K_{N}^{\perp} / H_{N}^{\perp} \subset K_{n}^{\perp} / H_{N}^{\perp}$ : Let $x \in H_{N}, \gamma \in K_{N}^{\perp}$. Then $\left\langle x+K_{N}, \gamma+H_{N}^{\perp}\right\rangle_{N N}=\left\langle x+K_{n}, \gamma+H_{N}^{\perp}\right\rangle_{N n}=\left\langle x+K_{n}, \gamma+H_{n}^{\perp}\right\rangle_{n n}$ from (8). Thus, the dual of the inclusion $H_{N} / K_{n} \subset H_{n} / K_{n}$ is the natural map $K_{n}^{\perp} / H_{N}^{\perp} \longleftarrow K_{n}^{\perp} / H_{n}^{\perp}$, and the other way around for the original natural map. As the standard dual pairing in particular is of the form (4), we have in the dual basis,

$$
\widehat{i_{N n}}=\left(\begin{array}{ccc}
\text { id } & & \\
& \widehat{\theta_{N}^{n}} & \\
& \widehat{\phi_{N}^{n}} & \widehat{\iota_{N}^{n}}
\end{array}\right) \text { and } \widehat{j_{N n}}=\left(\begin{array}{ccc}
\widehat{\kappa_{N}^{n}} & & \\
\widehat{\phi_{N}^{\prime n}} & \widehat{\theta_{N}^{\prime n}} & \\
& & \text { id }
\end{array}\right) \text {. }
$$

The meaning of $\widehat{\theta_{N}^{n}}$ etc. is clear from the definition of the standard dual pairing.

The dual map of $\kappa_{N}^{n}, \widehat{\kappa_{N}^{n}}$, is an injection $\mathbf{Z}^{a_{N}} \widehat{\kappa_{N}^{n}} \longrightarrow \mathbf{Z}^{a_{n}}$. The form of the standard dual pairing shows that $\widehat{\kappa_{N}^{n}}\left(\mathbf{Z}^{a_{N}}\right) \subset \mathbf{Z}^{a_{N}} \oplus\{0\}^{a_{n}-a_{N}}$. The maps $\left\{\iota_{N}^{n}\right\}$ and $\left\{\widehat{\kappa_{N}^{n}}\right\}$ are used in the definitions of the next section.

3.3. The inclusion $L^{2}\left(H_{N} / K_{n}\right) \longrightarrow L^{2}\left(G^{1}\right)$. Later, we apply the structure theory on the level of functions. Let us still work with $G^{1}$. As $H_{N} / K_{n}$ is needed to describe the relation between $H_{n} / K_{n}$ and $H_{N} / K_{N}$, we incorporate $H_{N} / K_{n}$ in the analysis of this situation. Define for any positive integers $N, n$ the linear map

$$
L^{2}\left(H_{N} / K_{n}\right) \stackrel{S_{N}^{n}}{\longrightarrow} L^{2}\left(G^{1}\right) \quad \text { by } \quad\left(S_{N}^{n} \Phi\right)(x)=\left\{\begin{array}{ll}
\Phi\left(x+K_{n}\right) & \text { if } x \in H_{N} \\
0 & \text { otherwise }
\end{array} .\right.
$$

The norm of $S_{N}^{n} \Phi$ is finite as $H_{N}$ is an open subgroup and $K_{n}$ is a compact subgroup (explained in Paragraph 3.3.1).

Let $G^{1}$ have some fixed Haar measure. Then there is a unique Haar measure on $H_{N} / K_{n}$ such that $S_{N}^{n}$ is an isometry. It turns out that we do not need to know more about these measures for the main approximation result, Section 6. Nevertheless, the next paragraph gives an explicit description of these measures, and for convenience we will use these measures in the rest of this paper. To simplify the notation we set $S_{n}:=S_{n}^{n}$.

3.3.1. Measures. If $B$ is a subgroup of the abelian group $A$, we say that $(A, B, A / B)$ is a Weil triple if $A, B$, and $A / B$ have Haar measures satisfying Weil formula, symbolically written $d_{A / B} \cdot d_{B}=d_{A}$. If $K$ is a compact group, by normalized measure, we mean the Haar measure on $K$ such that total measure of $K$ is one.

We make the following choice: $H_{N}$ has restricted measure as an open 
subgroup of $G^{1}, K_{n}$ has normalized measure, and $\left(H_{N}, K_{n}, H_{N} / K_{n}\right)$ is a Weil triple. This defines the correct measure on $H_{N} / K_{n}$ :

$$
\begin{aligned}
\int_{G^{1}}\left|\left(S_{N}^{n} f\right)(x)\right|^{2} d x & =\int_{H_{N}}\left|\left(S_{N}^{n} f\right)(x)\right|^{2} d x \\
& =\int_{H_{N} / K_{n}}\left|f\left(x+K_{n}\right)\right|^{2} d\left(x+K_{n}\right) \cdot \operatorname{meas}_{K_{n}}\left(K_{n}\right) \\
& =\int_{H_{N} / K_{n}}\left|f\left(x+K_{n}\right)\right|^{2} d\left(x+K_{n}\right) .
\end{aligned}
$$

We omit the proof of the next lemma as this description is not strictly necessary for Theorem 6.1. By counting measure, we mean counting measure with point weight one.

Lemma 3.8. Let $G^{1}$ be a $G^{1}$-group with structure $\left\{\left(H_{N}, K_{n}\right)\right\}, H_{N} / K_{n} \simeq$ $E_{N n}=\mathrm{T}^{a_{n}} \oplus \mathrm{F}_{N}^{n} \oplus \mathbf{Z}^{b_{N}}$, and $\mathrm{F}_{n}^{n}=: \mathrm{F}_{n}$.

i) There is a Haar measure on $G^{1}$ such that the map $S_{1}$ is isometric when $E_{1}$ has the product measure where $\mathrm{Z}^{b_{1}}$ has counting measure, and both $\mathrm{T}^{a_{1}}$, and $\mathrm{F}_{1}$ have normalized measures.

ii) If $G^{1}$ has the measure of i), for any positive integer $n, S_{n}$ is isometric when $E_{n}$ has the product measure where $\mathrm{Z}^{b_{n}}$ has counting measure, and $\mathrm{T}^{a_{n}}$ has normalized measure. The measure on $\mathrm{F}_{n}$ has the same point weight as the point weight on $\mathrm{F}_{1}^{n}$ when $\mathrm{F}_{1}^{n}$ has normalized measure.

Part ii) of this lemma is illustrated in the examples of Section 7.

\section{Setup}

Let $G=\mathrm{R}^{e} \oplus G^{1}$, where $G^{1}$ is a second countable $G^{1}$-group. Recall Definition 2.1. The purpose of this section is to define finite approximands for $(U, V)$ on $G$. Because $G$ is a finite direct sum, we use the construction in Proposition 2.3. We first introduce some notation which will be explained below. Here, $n$ is an odd positive integer throughout this section, and for any such odd positive integer we define $n^{\circ}$ through $n=2 n^{\circ}+1$ :

$$
\begin{aligned}
\text { Finite abelian group } & G_{n}=Z_{n}^{e} \oplus G_{n}^{1}, \\
\text { Isometry } & I_{n}=R_{n}^{e} \otimes I_{n}^{1}, \\
\text { Group element } & y^{n}=\left(r^{n}, x^{n}\right) \in G_{n}, \\
\text { Dual group element } & \beta^{n}=\left(d^{n}, \beta^{n}\right) \in \widehat{G}_{n}=Z_{n}^{e} \oplus \widehat{G}_{n}^{1} .
\end{aligned}
$$

For an odd positive integer $j, Z_{j}=\left\{-j^{\circ},-j^{\circ}+1, \ldots,-1,0,1, \ldots, j^{\circ}\right\}$, considered as a finite cyclic group. The $e$-th power of $Z_{j}$ is denoted by $Z_{j}^{e}$. Moreover, self-duality is set up with $\langle k, l\rangle=e^{2 \pi i k l / j}$ for $k, l \in \mathbf{Z}_{j}$. 
4.1. Schwinger embedding. The real part $\mathrm{R}^{e}$ is handled by the groups and scalings $\epsilon_{n}$ of Schwinger [9]. We follow Schwinger as we use $\langle x, y\rangle=e^{i x y}$ for $x, y \in \mathrm{R}$. Then we get the same scalings $\epsilon_{n}$ as he used. The finite group has already been taken as $\mathbf{Z}_{n}^{e}$.

4.1.1. The maps $R_{n}^{e}$. Let $R_{n}^{e}: l^{2}\left(Z_{n}^{e}\right) \longrightarrow L^{2}\left(\mathrm{R}^{e}\right)$ be the $e$ times tensor map of the operator $R_{n}: l^{2}\left(\mathbf{Z}_{n}\right) \longrightarrow L^{2}(\mathrm{R}), \quad$ where for $k \in \mathbf{Z}_{n}, \quad \mathbf{I}_{\{k\}} \longrightarrow$ $\left(\epsilon_{n}\right)^{-1 / 2} \mathbf{I}_{\left[(k-1 / 2) \epsilon_{n},(k+1 / 2) \epsilon_{n}\right)}=\left(\epsilon_{n}\right)^{-1 / 2} \mathbf{I}_{I_{n}^{k}}$, where $\epsilon_{n}=\sqrt{2 \pi / n}$. Characteristic function for the Borel set $E$ is denoted by $I_{E}$.

4.1.2. Group element $r^{n}$ and dual group element $d^{n}$. Let $|\cdot|_{\infty}$ denote the supnorm $|r|_{\infty}=\max _{i=1, \ldots, j}\left|r_{i}\right|$ for $r=\left(r_{i}\right)$. For $r=\left(r_{i}\right) \in \mathrm{R}^{e}$, we approximate by $r^{n} \in \mathbf{Z}_{n}^{e}$ in the following way: If $|r|_{\infty} \leq\left(n^{\circ}+1 / 2\right) \epsilon_{n}$, then define $r^{n}=\left(r_{1}^{n}, \ldots, r_{e}^{n}\right) \in \mathbf{Z}_{n}^{e}$, where $r_{i}^{n}$ is the unique integer such that $r_{i} \in\left[\left(r_{i}^{n}-1 / 2\right) \epsilon_{n},\left(r_{i}^{n}+1 / 2\right) \epsilon_{n}\right)$. Otherwise, $r^{n}$ is by definition 0 . We identify $\mathrm{R}^{e}$ and its dual group, and the approximation of $d \in \mathrm{R}^{e}$ is given by the same procedure as that for $r$.

We turn to the $G^{1}$-group part of the set up. Here, the idea is to use the structure theory of the previous section to get hold of finite abelian groups $G_{n}^{1}$. This set up will relate to the standard duality (7).

4.2. The groups $G_{n}^{1}$. Let $\mathrm{F}_{k m n}, k, m, n$ odd positive integers, be given by $\mathrm{F}_{k m n}=\mathbf{Z}_{m}^{a_{n}} \oplus \mathbf{F}_{n} \oplus \mathbf{Z}_{k}^{b_{n}}$. The numbers $b_{n}, a_{n}$ and the groups $\mathrm{F}_{n}$ come from the elementary group structure of $G^{1}$, Proposition 3.2; $H_{n} / K_{n} \simeq$ $E_{n}=\mathrm{T}^{a_{n}} \oplus \mathrm{F}_{n} \oplus \mathbf{Z}^{b_{n}}$ for some choice of $\left\{H_{n}\right\}$ and $\left\{K_{n}\right\}$. For $j$ odd, let $F_{j}^{b}$ consist of those $k \in \mathbf{Z}^{b}$ for which $|k|_{\infty} \leq j^{\circ}$, the $j$-cube in $\mathbf{Z}^{b}$ centered in origo. Recall that we in Section 3 found $\iota_{i}^{n}: \mathbb{Z}^{b_{i}} \longrightarrow \mathbf{Z}^{b_{n}}$ for $i<n$. Let $k_{n}$ be the smallest odd integer such that $\iota_{i}^{n}\left(F_{n}^{b_{i}}\right) \subset F_{k_{n}}^{b_{n}}$ for all $i<n$. Equivalently, $k_{n}$ is the smallest odd integer such that $|k|_{\infty} \leq n^{\circ}$ implies $\left|\iota_{i}^{n}(k)\right|_{\infty} \leq k_{n}^{\circ}$ for any $i<n$. Likewise, as $\widehat{\kappa_{i}^{n}}: \mathbf{Z}^{a_{i}} \longrightarrow \mathbf{Z}^{a_{n}}$ for $i<n$, let $m_{n}$ be the smallest odd integer such that $\widehat{\kappa}_{i}^{n}\left(F_{n}^{a_{i}}\right) \subset F_{m_{n}}^{a_{n}}$ for all $i<n$. For $n$ odd define $G_{n}^{1}:=\mathrm{F}_{k_{n} m_{n} n}=\mathbf{Z}_{m_{n}}^{a_{n}} \oplus \mathrm{F}_{n} \oplus \mathbf{Z}_{k_{n}}^{b_{n}}$.

4.3. The embeddings $I_{n}^{1}$. We start by constructing $I_{k m n}: l^{2}\left(\mathrm{~F}_{k m n}\right) \longrightarrow L^{2}\left(G^{1}\right)$. These maps are defined through

$$
\begin{aligned}
l^{2}\left(\mathrm{~F}_{k m n}\right) & \simeq l^{2}\left(\mathrm{Z}_{m}^{a_{n}}\right) \otimes l^{2}\left(\mathrm{~F}_{n}\right) \otimes l^{2}\left(\mathrm{Z}_{k}^{b_{n}}\right) \\
& \stackrel{T_{m}^{a_{n}} \otimes \mathrm{i} \otimes \mathrm{Z}_{\mathrm{k}}^{\mathrm{b}_{n}}}{\longrightarrow} \\
L & L^{2}\left(\mathrm{~T}^{a_{n}}\right) \otimes l^{2}\left(\mathrm{~F}_{n}\right) \otimes l^{2}\left(\mathbf{Z}^{b_{n}}\right) \simeq L^{2}\left(H_{n} / K_{n}\right) \stackrel{S_{n}}{\longrightarrow} L^{2}\left(G^{1}\right) .
\end{aligned}
$$

Here we identify $I_{k m n}$ with $S_{n}\left(T_{m}^{a_{n}} \otimes \mathrm{id} \otimes Z_{k}^{b_{n}}\right), S_{n}$ is the lift of Paragraph 3.3. Finally, let $I_{n}^{1}:=I_{k_{n} m_{n} n}$.

The measures on $Z^{b_{n}}, \mathrm{~T}^{a_{n}}$, and $\mathrm{F}_{n}$ are those of Lemma 3.8. The maps $Z_{k}^{b_{n}}$ 
and $T_{m}^{a_{n}}$ are defined below in order to be isometric when $Z_{k}^{b_{n}}$ has the usual counting measure, while $Z_{m}^{a_{n}}$ has normalized measure.

4.3.1. The maps $Z_{k}^{b_{n}}$. The embeddings $Z_{k}^{b_{n}}: l^{2}\left(Z_{k}^{b_{n}}\right) \longrightarrow l^{2}\left(Z^{b_{n}}\right)$ are defined in the obvious way by sending $\mathrm{I}_{\{i\}}$ to $\mathrm{I}_{\{i\}}$ in $l^{2}\left(\mathbf{Z}^{b_{n}}\right)$ for $i \in \mathbf{Z}_{k}^{b_{n}}$.

4.3.2. The maps $T_{m}^{a_{n}}$. Let $T_{m}^{a_{n}}=\mathscr{F}^{n} Z_{m}^{a_{n}}\left(\mathscr{F}_{m n}\right)^{-1}$, where $\mathscr{F}_{m n}: l^{2}\left(Z_{m}^{a_{n}}\right) \longrightarrow$ $l^{2}\left(\mathbf{Z}_{m}^{a_{n}}\right)$ and $\mathscr{F}^{n}: l^{2}\left(\mathbf{Z}^{a_{n}}\right) \longrightarrow L^{2}\left(\mathbf{T}^{a_{n}}\right)$ are Fourier transforms. This is a variation of the approach in Proposition 2.2.

4.4. Approximate group element in $G^{1}$. Given $x \in G^{1}$, we associate to it $x^{k m n} \in \mathrm{F}_{k m n}$. As $G^{1}=\cup H_{n}, x \in H_{W_{x}}$ for some smallest integer $W_{x}$. For $n \geq W_{x}$, surject (recall Equation 8) $x \longrightarrow x+K_{n}=x_{n}=\left(t_{n}, f_{n}, z_{n}\right) \in E_{n}$. Then let $x^{k m n}=\left(t^{m n}, f_{n}, z^{k n}\right)$ if $n \geq W_{x}$, and 0 otherwise, where the elements $z^{k n} \in Z_{k}^{b_{n}}$ and $t^{m n} \in Z_{m}^{a_{n}}$ are defined below. Finally, let $x^{n}:=x^{k_{n} m_{n} n}, t^{n}:=t^{m_{n} n}$ and $z^{n}:=z^{k_{n} n}$.

4.4.1. The integer part $z^{k n}$. If $z_{n} \in F_{k}^{b_{n}}$, let $z_{n}=: z^{k n} \in \mathbf{Z}_{k}^{b_{n}}$. Otherwise, put $z^{k n}$ to 0 .

4.4.2. The circle part $t^{m n}$. Here we apply root functions. Parametrize the circle $\mathrm{T}$ by $z=e^{2 \pi i \theta}, \quad \theta \in[-1 / 2,1 / 2)$. For our $t_{n}=\left(t_{n, j}\right) \in \mathrm{T}^{a_{n}}$, let $t^{m n}=\left(t_{j}^{m n}\right) \in \mathbf{Z}_{m}^{a_{n}}$, where $t_{j}^{m n}$ is the unique element in $\mathbf{Z}_{m}$ such that $\theta_{n, j} \in\left[\left(t_{j}^{m n}-1 / 2\right) / m,\left(t_{j}^{m n}+1 / 2\right) / m\right)$ for $t_{n, j}=e^{2 \pi i \theta_{n, j}}$.

4.5. Approximate character in $\widehat{G^{1}}$. Recall the dual construction and definitions of Paragraph 3.2. Therefore, for $\gamma \in \widehat{G^{1}}, \gamma^{k m n} \in \mathbf{Z}_{m}^{a_{n}} \oplus \mathbf{F}_{n} \oplus \mathbf{Z}_{k}^{b_{n}}$ (dual basis) is chosen by the same procedure as for the group element case. Let us fix some notation: For $n \geq \hat{W}_{\gamma} \quad\left(\hat{W}_{\gamma}\right.$ chosen analogous to $\left.W_{x}\right)$, $\gamma_{n}=\left(u_{n}, g_{n}, s_{n}\right) \in \widehat{E_{n}}$ (recall Equation 9). Then $\gamma^{k m n}=\left(u^{m n}, g_{n}, s^{k n}\right)$ if $n \geq \hat{W}_{\gamma}$, and 0 otherwise. Here the circle part $s^{k n} \in \mathbf{Z}_{k}^{b_{n}}$ and the integer part $u^{m n} \in \mathbf{Z}_{m}^{a_{n}}$ are defined by the procedures in the last paragraph (with reverse notation). Finally, we set $\gamma^{n}:=\gamma^{k_{n} m_{n} n}, u^{n}:=u^{m_{n} n}$ and $s^{n}:=s^{k_{n} n}$.

\section{The Space of Schwartz-Bruhat Functions}

Let $G$ be a 1.c.a. group. Recall from Section 3 the existence of pairs $(H, K)$, where $H / K$ is elementary, $H$ is an open subgroup, and $K \subset H$ is a compact subgroup.

Bruhat [1] introduces the Schwartz-Bruhat space of functions on $G, \mathscr{S}(G)$, as those complex valued functions which have support in some $H$, and are locally constant on some corresponding $K$. Thus $\Phi$ is naturally defined on the elementary group $H / K$, here $\Phi$ should look like an ordinary Schwartz function. This means: Let $P$ be a polynomial function on $H / K$, and $D$ a 
translation invariant differential operator. Then $\Phi \in \mathscr{S}(H / K)$ if $\Phi$ is smooth, and all the seminorms $\|P \cdot D f\|_{\infty}$ are finite. Alternatively, this can be formulated by the tensor product of Grothendieck for locally convex spaces (Schwartz spaces are nuclear). We use $T_{H K}$ to denote

$$
\begin{aligned}
& \Phi \in L^{2}\left(G^{1}\right) \longrightarrow T_{H K} \Phi \in L^{2}(H / K),\left(T_{H K} \Phi\right)(x+K)=\Phi(x), \\
& x \in x+K \in H / K .
\end{aligned}
$$

Notice that $T_{H K}$ is the inverse of $S_{N}^{n}$ (Paragraph 3.3) for $H=H_{N}$ and $K=K_{n}$ on the range of $S_{N}^{n}$. If $H \subset H^{\prime}$ and $K^{\prime} \subset K$, then $\Phi$ Schwartz-Bruhat on $(H, K)$ implies $\Phi$ Schwartz-Bruhat on $\left(H^{\prime}, K^{\prime}\right)$ as well. There are "large" enough pairs $(H, K)$ for $\mathscr{S}(G)$ to be dense in $L^{2}(G)$, and the Fourier transform leaves this space invariant; if $\Phi$ is Schwartz-Bruhat on $(H, K)$, then $\hat{\Phi}$ is Schwartz-Bruhat on $\left(K^{\perp}, H^{\perp}\right)$.

As $G$ by [11] is of the form $\mathrm{R}^{e} \oplus G^{1}$, where $G^{1}$ is a $G^{1}$-group, the definition of $\mathscr{S}\left(G^{1}\right)$ is really what is new in this extension of Schwartz functions.

Let $G^{1}$ be a second countable $G^{1}$-group. Use Proposition 3.2 to find $\left\{H_{N}\right\}$, $\left\{K_{n}\right\}$. It suffices to define $\mathscr{S}\left(G^{1}\right)$ on the pairs $\left\{\left(H_{n}, K_{n}\right)\right\}$ :

Lemma 5.1. Let $G^{1},\left\{H_{n}\right\}$ and $\left\{K_{n}\right\}$ satisfy the conclusions of Proposition 3.2. If $(H, K)$ is some other pair in the definition of $\mathscr{S}\left(G^{1}\right)$, then there is a nonnegative integer $n$ such that $H_{n} \supset H$ and $K_{n} \subset K$.

Proof. First, $H / K \simeq \mathbf{T}^{a} \oplus \mathbf{F} \oplus \mathbf{Z}^{b}$, so, as the quotient is compactly generated and $K$ is compact, $H$ itself is compactly generated (the pre-image of a compact set is compact as $K$ is compact). If $C$ is compact and generates $H$, then $G^{1}=\cup H_{\eta}$ covers $C$, and $C \subset H_{N}$ for some integer $N$. Thus, $H \subset H_{N}$. Next, $K^{\perp} \subset \widehat{G^{1}}$ is open while $H^{\perp} \subset K^{\perp} \subset \widehat{G^{1}}$ is compact. Moreover, $K^{\perp} / H^{\perp} \simeq \widehat{H / K}$, so $K^{\perp}$ is compactly generated, and the same reasoning as before gives $K^{\perp} \subset K_{M}^{\perp}$ and $K_{M} \subset K$. So the claim follows with $n$ as the larger of $N$ and $M$.

We use $\Phi \in \mathscr{S}(n)$ to denote $\Phi \in \mathscr{S}\left(G^{1}\right)$ supported in $H_{n} / K_{n}$. Notice that $\mathscr{S}(n) \subset \mathscr{S}\left(n^{\prime}\right)$ when $n<n^{\prime}$. Also, put $\Phi^{n}:=T_{H_{n} K_{n}} \Phi$ for $\Phi \in \mathscr{S}(n)$. Let $V^{n}$ and $U^{n}$ denote the standard Weyl system on $L^{2}\left(H_{n} / K_{n}\right)$.

Lemma 5.2. Assume $x \in G^{1}, \gamma \in \widehat{G^{1}}$ and $\Phi \in \mathscr{S}\left(G^{1}\right)$. Then we can find an $n$ such that $\Phi, \quad V(x) \Phi$ and $U(\gamma) \Phi$ are all contained in $\mathscr{S}(n)$, $(V(x) \Phi)^{n}=V^{n}\left(x+K_{n}\right) \Phi^{n}$, and $(U(\gamma) \Phi)^{n}=U^{n}\left(\gamma+H_{n}^{\perp}\right) \Phi^{n}$.

Proof. Assume $\Phi \in \mathscr{S}\left(n^{\prime}\right)$. Since $H_{n} \nearrow G^{1}, x$ is contained in some $H_{n^{\prime \prime}}$, and by the group property of any $H_{n}$ it follows that $V(x) \Phi \in \mathscr{S}\left(\max \left\{n^{\prime}, n^{\prime \prime}\right\}\right)$. Replacing $x$ by $x+k$, for $k \in K_{n}$, clearly makes no difference. That multiplication by character is locally constant is a special 
case of Weil [16], Proposition 2. Here, the proof is straightforward: As $\cup K_{n}^{\perp}=\widehat{G^{1}}, \gamma \in K_{n}^{\perp}$ for some $n$. Thus, multiplication by $\gamma$ is automatically locally constant on $K_{n}$. Moreover, since $\Phi$ is supported in $H_{n}$, multiplication by $\gamma$ or $\gamma+\gamma^{\prime}$, for $\gamma^{\prime} \in H_{n}^{\perp}$, gives the same result. The largest $n$ from the two parts of the proof gives the desired result as translation and multiplication by character is invariant for the Schwartz space of any elementary group.

Using $I_{n}=S_{n}$, it follows easily from this lemma that $G^{1}$ is a limit of the elementary groups $H_{n} / K_{n}$ in the sense of Weyl systems.

5.1. Exploring $\mathscr{S}\left(G^{1}\right)$. Assume that $G^{1}$ is a second countable $G^{1}$-group. For $n>N$, if $\Phi \in \mathscr{S}(N)$, then $\Phi \in \mathscr{S}(n)$. We need more on the relationship between $\Phi^{n}$ and $\Phi^{N}$. Recall from Section 3 the induced maps $E_{N} \stackrel{j_{N n}}{\longleftarrow} E_{N n} \stackrel{i_{N n}}{\longrightarrow} E_{n}$. From their construction,

$$
\Phi^{n}=\left\{\begin{array}{ll}
\Phi^{N} \circ j_{N n} \circ\left(i_{N n}\right)^{-1} & \text { on } i_{N n}\left(E_{N n}\right) \\
0 & \text { otherwise. }
\end{array} .\right.
$$

We easily get this by passing through $H_{N} / K_{n}$. Notice that scaling factors would enter without the measure considerations of Paragraph 3.3.1. Let $\Phi \in \mathscr{S}(N)$, where in addition $\Phi^{N}=\Phi_{\mathrm{T}}^{N} \Phi_{\mathrm{F}}^{N} \Phi_{\mathrm{Z}}^{N}$ is a simple tensor; $\Phi_{\mathrm{T}}^{N} \in \mathscr{S}\left(\mathrm{T}^{a_{N}}\right), \Phi_{\mathrm{F}}^{N} \in \mathscr{S}\left(\mathrm{F}_{N}\right)$, and $\Phi_{\mathrm{Z}}^{N} \in \mathscr{S}\left(\mathrm{Z}^{b_{N}}\right)$. Then by Proposition 3.7, for $(t, f, z) \in E_{n}=\mathrm{T}^{a_{n}} \oplus \mathrm{F}_{n} \oplus \mathbf{Z}^{b_{n}}$ and $n>N$ (primed coordinates are in $E_{N n}$ ),

$$
\Phi^{n}(t, f, z)= \begin{cases}\Phi_{\mathrm{\top}}^{N}\left(\kappa_{N}^{n}\left(T 0+\phi_{N}^{\prime n}\left(f^{\prime}\right)\right)\right. & \text { if } z=\iota_{N}^{n}\left(z^{\prime}\right) \text { and } f=\phi_{n}^{n}\left(z^{\prime}\right)+\theta_{n}^{n}\left(f^{\prime}\right) \\ 0 & \text { otherwise }\end{cases}
$$

$$
\begin{aligned}
& \times \begin{cases}\Phi_{\mathrm{F}}^{N}\left(\theta_{N}^{\prime n}\left(f^{\prime}\right)\right) & \text { if } z=\iota_{N}^{N}\left(z^{\prime}\right) \text { and } f=\phi_{n}^{n}\left(z^{\prime}\right)+\theta_{N}^{n}\left(f^{\prime}\right) \\
0 & \text { otherwise }\end{cases} \\
& \times\left\{\begin{array}{ll}
\Phi_{\mathrm{Z}}^{n}\left(z^{\prime}\right) & \text { if } z=\iota_{N}^{n}\left(z^{\prime}\right) \\
0 & \text { otherwise }
\end{array} .\right.
\end{aligned}
$$

This is a product of one function in all three coordinates, one function in $F_{n}$ and $Z^{b_{n}}$, and one function in $Z^{b_{n}}$ alone. We see the same by calculating $j_{N n} \circ i_{N n}^{-1}$ (on the image of $i_{N n}$ ). This matrix is upper triangular.

Recall the Fourier transform $\mathscr{F}^{n}$ on $\mathrm{Z}^{a_{n}}$. Let $D_{n}=\mathrm{Z}^{a_{n}} \oplus \mathrm{F}_{n} \oplus \mathrm{Z}^{b_{n}}$. We also denote $\mathscr{F}^{n} \otimes \mathrm{id} \otimes \mathrm{id}$ acting on $l^{2}\left(D_{n}\right)$ by $\mathscr{F}^{n}$. We generally skip all $\otimes$ and any id in the notation for tensor product of operators. So, for instance, we use the same symbol for an operator and its amplification by identity operators.

Lemma 5.3. Let $\Psi_{\mathrm{\top}}^{N}=\left(\mathscr{F}^{N}\right)^{-1} \Phi_{\mathrm{\top}}^{N}, n$ any integer larger than $N$, and $\Psi^{n}=\left(\mathscr{F}^{n}\right)^{-1} \Phi^{n} \in l^{2}\left(D_{n}\right)$. Then 


$$
\Psi^{n}=\Psi_{\mathrm{T}}^{n} \cdot \Lambda_{n} \cdot \Phi_{\mathrm{Z}}^{n},
$$

where $\Psi_{\mathrm{T}}^{n}=\Psi_{\mathrm{T}}^{N} \circ\left[\widehat{\kappa_{N}^{n}}\right]^{-1}$ (and 0 outside range of $\widehat{\kappa_{N}^{n}}$ ) is a function of $Z^{a_{n}}$, $\Phi_{\mathrm{Z}}^{n}=\Phi_{\mathrm{Z}}^{N} \circ\left[\iota_{N}^{n}\right]^{-1}$ (and 0 outside range of $\iota_{N}^{n}$ ) is a function of $\mathrm{Z}^{b_{n}}$, while $\Lambda_{n}$ depends on all three coordinates.

Proof. From (5.1) only the first part in the product formula for $\Phi$, $\Xi:=\Phi_{\mathrm{\top}}\left(\kappa_{N} n(t)+\phi_{N}^{\prime n}\left(f^{\prime}\right)\right)$ if $z=\iota_{N}^{n}\left(z^{\prime}\right), f=\phi_{N}^{n}\left(z^{\prime}\right)+\theta_{N}^{n}\left(f^{\prime}\right)$, and 0 otherwise, depends on $\mathrm{T}^{a_{n}}$. Let $q=(k, f, z) \in D_{n}$. First, if $f$ and/or $z$ is outside the range of $i_{N n}, i^{n}$ is obviously 0 . This settles the ,otherwise" case for the requirements for $f$ and $z$. If not, we are left with Fourier transform in $\mathrm{T}^{a_{n}}$-direction of the function $\Phi_{\top}^{N}\left(\kappa_{n}^{N}(t)+F_{N}^{n}(z, f)\right)$, where $F_{N}^{n}(z, f):=\phi_{N}^{\prime n}\left(f^{\prime}\right)$ to show clearer that $f^{\prime}$ also depends on $z$. Thus, we take the inverse Fourier transform of a translated function that is locally constant on $\operatorname{Ker}\left(\kappa_{N}^{n}\right)$. Then the Weil formula shows that the Fourier transform is supported in the range of $\widehat{\kappa_{N}^{n}}$, and translation goes to multiplication:

$$
\begin{aligned}
\left(\left(\mathscr{F}^{n}\right)^{-} 1 \Xi^{n}\right)(q) & =\int_{t \in T_{n}^{a}} \Phi_{\mathrm{T}}^{N}\left(\kappa_{N}^{n}(t)+F_{N}^{n}(f, z)\right)\langle k, t\rangle d t \\
& =\int_{\in T_{n}^{a} / \kappa_{N}^{n}} \Phi_{\mathrm{T}}^{N}\left(\kappa_{N}^{n}(\tilde{t})+F_{N}^{n}(f, z)\right)\left\langle k, \tilde{t} \int_{t^{\prime} \in \kappa_{N}^{n}}\left\langle k, t^{\prime}\right\rangle d t^{\prime} .\right.
\end{aligned}
$$

In the last line, $\kappa_{N}^{n}$ also denotes the map (isomorphism) from the quotient $\mathrm{T}^{a_{n}} / \operatorname{Ker} \kappa_{N}^{n}$ to $\mathrm{T}^{a_{N}}$. From Paragraph 3.3.1, $\operatorname{Ker}\left(\kappa_{N}^{n}\right)$ has normalized measure as this compact group is the circle part of $K_{N} / K_{n}$. Then as $\mathrm{T}^{a_{n}}$ has normalized measure, also the quotient $\mathrm{T}^{a_{N}}$ has normalized measure. The integral in $t^{\prime}$ is non-zero, and then it always has value 1 , if and only if $k$ is in the annihilator of $\operatorname{Ker}\left(\kappa_{N}^{n}\right)$, which means that $k \in \operatorname{Im}\left(\widehat{\kappa_{N}^{n}}\right)$. That takes care of rest of the 'otherwise' claims, and gives for $k=\widehat{\kappa_{N}^{n}}\left(k^{\prime}\right)$

$$
\begin{aligned}
\left(\left(\mathscr{\mathscr { F }}^{n}\right)^{-1} \Xi^{n}\right)(q) & =\int_{\tilde{t}} \Phi_{\mathrm{T}}^{N}\left(\kappa_{N}^{n}(\tilde{t})+F_{N}^{n}(f, z)\right)\left\langle\widehat{\kappa_{N}^{n}}\left(k^{\prime}\right), \tilde{t}\right\rangle d \tilde{t} \\
& =\int_{\tilde{t}} \Phi_{\mathrm{\top}}^{N}\left(\kappa_{N}^{n}(\tilde{t})+F_{N}^{n}(f, z)\right)\left\langle k^{\prime}, \kappa_{N}^{n}(\tilde{t})\right\rangle d \tilde{t} \\
& =\int_{u \in T_{N}^{a}} \Phi_{\mathrm{\top}}^{N}(u)\left\langle k^{\prime}, u\right\rangle d u \cdot\left\langle-k^{\prime}, F_{N}^{n}(f, z)\right\rangle \\
& =\Psi_{\mathrm{T}}^{n}\left(k^{\prime}\right) \cdot\left\langle-k^{\prime}, F_{N}^{n}(f, z)\right\rangle .
\end{aligned}
$$

Combined with (11), the result follows.

Without the measure considerations of Paragraph 3.3.1, again scaling factors enter (but can be taken into $\Lambda_{n}$ ). 
This lemma has the following important consequence: Let Supp denote the support of a function, then Lemma 5.3 shows that Supp $\Psi^{n} \subset$ $\widehat{\kappa_{N}^{n}}\left(\operatorname{Supp} \Psi_{\mathrm{T}}^{N}\right) \oplus \mathrm{F}_{n} \oplus \iota_{N}^{n}\left(\operatorname{Supp} \Phi_{\mathrm{Z}}^{N}\right)$. This motivates the choice of the size parameters $k_{n}$ and $m_{n}$ in Section 4 .

\section{Main Approximation Result}

THEOREM 6.1. The second countable locally compact abelian group $G$ is a limit, in the sense of Weyl systems (Definition 2.1), of the finite abelian groups $\left\{G_{n}\right\}_{n \text { odd }}$ defined in Section 4.

Proof. Propositions 2.3, 3.2, and Propositions 6.3, 6.4 below.

6.1. The $G^{1}$-case.

Proposition 6.2. Let $x \in G^{1}$, and $\gamma \in \widehat{G^{1}}$. Then $\left\langle x^{n}, \gamma^{n}\right\rangle_{G_{n}^{1}} \longrightarrow\langle x, \gamma\rangle_{G^{1}}$ as $n \longrightarrow \infty(n$ odd $)$. Here, $x^{n} \in G_{n}^{1}$ and $\gamma^{n} \in \widehat{G_{n}^{1}}$ are as in Section 4 .

Proof. For $n \geq W$, the larger of $W_{x}$ and $\hat{W}_{\gamma}$ (as in Section 4), by Equation $12,\langle x, \gamma\rangle=\left\langle x+K_{n}, \gamma+H_{n}^{\perp}\right\rangle_{H_{n} / K_{n}}=\left\langle x_{n}, \gamma_{n}\right\rangle_{s}$. Thus, for $n \geq W$

$$
\begin{aligned}
\left|\left\langle x^{n}, \gamma^{n}\right\rangle_{G_{n}^{1}}-\langle x, \gamma\rangle_{G^{1}}\right|= & \left|\left\langle\left(t^{n}, f_{n}, z^{n}\right),\left(u^{n}, g_{n}, s^{n}\right)\right\rangle_{G_{n}^{1}}-\left\langle\left(t_{n}, f_{n}, z_{n}\right),\left(u_{n}, g_{n}, s_{n}\right)\right\rangle_{s}\right| \\
\leq & \left|\left\langle z^{n}, s^{n}\right\rangle-\left\langle z_{n}, s_{n}\right\rangle_{Z_{n}^{b}}\right| \\
& +\left|\left\langle t^{n}, u^{n}\right\rangle-\left\langle t_{n}, u_{n}\right\rangle_{T_{n}^{a}}\right| .
\end{aligned}
$$

For the inequality, write out the characters as products, then use triangle inequalities, and the fact that all numbers involved have absolute value one.

Let us estimate (12). Here, $z_{n}=\iota_{W}^{n}\left(z_{W}\right)$ by semi-alignment Proposition 3.7. Observe that $k_{n}^{\circ} \geq\left|z_{n}\right|_{\infty}$ for all $n$ larger than some $L$ : Let $L$ be such that $\left|z_{W}\right|_{\infty} \leq n^{\circ}$ for $n \geq L$. Then by construction in Section $4,\left|\iota_{W}^{n}\left(z_{W}\right)\right|_{\infty} \leq k_{n}^{\circ}$. Consequently, for $n$ larger than $L$ (taken $>W$ ), $z^{n}=z_{n}=\iota_{W}^{n}\left(z_{W}\right)$. Again by Proposition 3.7, $\iota_{W}^{n}\left(Z^{b_{W}}\right)$ is contained in the first $b_{W}$ factors of $Z^{b_{n}}$. Thus $\left\langle z_{n}, s_{n}\right\rangle=\prod_{l=1}^{b_{W}} e^{2 \pi i i_{W}^{n}\left(z_{W}\right)_{l} \theta_{n, l}}$ where $s_{n}=\left(s_{n, l}\right), s_{n, l}=e^{2 \pi i \theta_{n, l}}$. Likewise, for this large $n,\left\langle z^{n}, s^{n}\right\rangle=\prod_{l=1}^{b_{W}} e^{2 \pi i u_{W}^{n}\left(z_{W}\right)_{l} \theta_{n, l}^{n}}$ where $s^{n}=\left(s_{l}^{n}\right), s_{l}^{n} / k_{n}=\theta_{n, l}^{n}$. Then we calculate

$$
(12) \leq \sum_{l=1}^{b_{W}}\left|1-e^{2 \pi i u_{W}^{n}\left(z_{W}\right)_{l} \delta_{n}^{l}}\right|
$$

as $\theta_{n, l}^{n}=\theta_{n, l}+\delta_{n}^{l}$ where $\left|\delta_{n}^{l}\right| \leq 1 /\left(2 k_{n}\right)$. Moreover, (12) tends to 0 because

$$
\left|\iota_{W}^{n}\left(z_{W}\right)_{l} \delta_{n}^{l}\right| \leq b_{W}\left|z_{W}\right|_{\infty} k_{n}^{\circ} /\left(n^{\circ} 2 k_{n}\right) \leq b_{W}\left|z_{W}\right|_{\infty} /\left(2 n^{\circ}\right) .
$$

The estimate on $\iota_{W}^{n}\left(z_{W}\right)_{l}$ comes from the fact that $\iota_{W}^{n}$ is a Z-module map 
between two free Z-modules. In the standard bases, $\iota_{W}^{n}$ has integer coefficient matrix representation $\left(a_{j}^{l}\right)$. By the construction of $k_{n}$, this is easily seen to imply $\left|a_{j}^{l}\right| \leq k_{n}^{\circ} / n^{\circ}$. Consequently, $\left|\iota_{W}^{n}\left(z_{n}\right)_{l}\right| \leq b_{W} \max _{j}\left|z_{W, j}\right| k_{n}^{\circ} / n^{\circ}$ follow from this matrix representation.

Finally, (13) is estimated as (12) because $m_{n}$ is constructed similar to $k_{n}$.

Let us settle some matters of notation for the projections of Section 4: $P_{n}: L^{2}\left(G^{1}\right) \rightarrow I_{n}^{1}\left(l^{2}\left(G_{n}^{1}\right)\right), \quad P_{n}^{\top}: L^{2}\left(\mathrm{~T}^{a_{n}}\right) \rightarrow T_{m_{n}}^{a_{n}}\left(l^{2}\left(\mathrm{Z}_{m_{n}}^{a_{n}}\right)\right)$, and $P_{n}^{\mathrm{Z}}: L^{2}\left(\mathrm{Z}^{b_{n}}\right) \rightarrow$ $Z_{k_{n}}^{b_{n}}\left(l^{2}\left(Z_{k_{n}}^{b_{n}}\right)\right)$.

Proposition 6.3. The second countable $G^{1}$-group $G^{1}$ is a limit, in the sense of Weyl systems, of the finite abelian groups $\left\{G_{n}^{1}\right\}_{n \text { odd }}$ of Section 4 .

Proof. Let $\Phi \in \mathscr{S}(N), N$ some positive integer, where $\Phi^{N}=\Phi_{\mathrm{T}}^{N} \Phi_{\mathrm{F}}^{N} \Phi_{\mathrm{Z}}^{N}$ is a simple tensor as in Paragraph 5.1. Moreover, $\Phi_{Z}^{N}$ is taken with finite support, and $\Phi_{\mathrm{T}}^{N}$ is a trigonometric polynomial.

The linear span of the chosen $\Phi$ is dense in $L^{2}\left(G^{1}\right)$ : Through $\Phi \longrightarrow \Phi^{N}$, the linear span of all the $L^{2}\left(E_{N}\right)$ is dense in $L^{2}\left(G^{1}\right)$. Moreover, it is easy to see that the linear span of simple tensors with finite support are dense in the discrete space $l^{2}\left(D_{N}\right)$. Applying $\mathscr{F}^{N}$ gives the desired density in $L^{2}\left(E_{N}\right)$ as the Fourier transform $\mathscr{F}^{N}$ on $Z^{a_{N}}$ takes finite support functions to trigonometric polynomials, and the other way around. Finally, the resulting functions are in $\mathscr{S}\left(E_{N}\right)$. Thus, we really work in a smaller space than $\mathscr{S}\left(G^{1}\right)$.

Convergence of projections. As $P_{n} \Phi \in \mathscr{S}(n),\left(P_{n} \Phi\right)^{n}=P_{n}^{\top} P_{n}^{\mathrm{Z}} \Phi^{n}$ from the definition of $P_{n}$ (the linear map $S_{n}$ is the inverse of $T_{H_{n} K_{n}}$ ), and

$$
\left\|P_{n} \Phi-\Phi\right\|_{G^{1}}=\left\|P_{n}^{\top} P_{n}^{\mathrm{Z}} \Phi^{n}-\Phi^{n}\right\|_{E_{n}}
$$

for $n>N$. Let $C^{n}=F_{m_{n}}^{a_{n}} \oplus \mathrm{F}_{n} \oplus F_{k_{n}}^{b_{n}}$. By the construction in Section 4 ( $\Psi^{n}$ is as in Paragraph 5.1), $P_{n}^{\top} P_{n}^{\mathrm{Z}} \Phi^{n}=\mathscr{F}^{n} \mathrm{I}_{C^{n}} \Psi^{n}$. As $\Phi_{\mathrm{Z}}^{N}$ and $\Psi_{\mathrm{T}}^{N}$ have finite support, there is a $Q$ (taken larger than $N$ ) such that for $n>Q, \operatorname{Supp}\left(\Phi_{\mathrm{Z}}^{N}\right) \subset F_{n}^{b_{N}}$ and $\operatorname{Supp}\left(\Psi_{\mathrm{T}}^{N}\right) \subset F_{n}^{a_{N}}$. Then, by definition of $k_{n}$ and $m_{n}$, and Lemma 5.3, $\operatorname{Supp}\left(\Psi^{n}\right) \subset C^{n}$. Thus, $P_{n} \Phi=\Phi$ for $n>Q$, and strong convergence of projection has been proven.

Convergence of the $V^{\prime}$ 's. Let $x \in G^{1}$ as in Proposition 6.2. Because of Lemma 5.2, for $n>M$, which is the larger of $N$ and $W$ ( $W$ as in Proposition 6.2), $\quad V(x) \Phi \in \mathscr{S}(n)$, and $(V(x) \Phi)^{n}=V^{n}\left(x_{n}\right) \Phi^{n}$. Here (Equation 8) $x_{n}=\left(t_{n}, f_{n}, z_{n}\right) \in E_{n}$. We agree on a notation where $V\left(V_{n}^{\prime}\right)$ denotes translation (finite embedded translation), and the argument tells us what group is involved. Then $(V(x) \Phi)^{n}=V\left(t_{n}\right) V\left(f_{n}\right) V\left(z_{n}\right) \Phi^{n}$ for $n>M$. Moreover, by construction of the isometry $S_{n}, \quad V_{n}^{\prime}\left(x^{n}\right) \Phi \in \mathscr{S}(n)$ for $n>N$, and $\left(V_{n}^{\prime}\left(x^{n}\right) \Phi\right)^{n}=V_{n}^{\prime}\left(t^{n}\right) V_{n}\left(f_{n}\right) V_{n}^{\prime}\left(z^{n}\right) \Phi^{n}$, where for instance $V_{n}^{\prime}\left(t^{n}\right)$ is the embedding of $V_{n}\left(t^{n}\right)$ through the map $T_{m_{n}}^{a_{n}}$. Thus, as $V\left(f_{n}\right)$ is unitary, for $n>M$, 


$$
\begin{aligned}
\left\|V(x) \Phi-V_{n}^{\prime}\left(x^{n}\right) \Phi\right\|_{G^{1}} & =\left\|V\left(z_{n}\right) V\left(t_{n}\right) \Phi^{n}-V_{n}^{\prime}\left(z^{n}\right) V_{n}^{\prime}\left(t^{n}\right) \Phi^{n}\right\|_{E_{n}} \\
& =\left\|V\left(z_{n}\right)\left(t_{n}\right) \Psi^{n}-V_{n}^{\prime}\left(z^{n}\right)_{n}^{\prime}\left(t^{n}\right) \Psi^{n}\right\|_{D_{n}} .
\end{aligned}
$$

From the definition in Section 4, $V_{n}^{\prime}\left(t^{n}\right)=\mathscr{F}^{n} \hat{U}_{n}^{\prime}\left(t^{n}\right)\left(\mathscr{F}^{n}\right)^{-1}$, where $\hat{U}_{n}\left(t^{n}\right)=U_{n}\left(t^{n}\right)$ as we identify $l^{2}\left(Z_{m_{n}}^{a_{n}}\right)$ with itself under the finite Fourier transform. Moreover, $\hat{U}\left(t_{n}\right)$ operates through multiplication by $\left\langle\cdot, t_{n}\right\rangle$ on $l^{2}\left(Z^{a_{n}}\right)$. Let $L$ (now taken $>M$ ) be so large that $z_{M} \subset F_{n}^{b_{M}}$ for $n>L$. Then from Section $4, z_{n}=\iota_{M}^{n}\left(z_{M}\right) \subset F_{k_{n}}^{b_{n}}$, and $z^{n}=z_{n}$. So, for $n>L$, the operator $V_{n}^{\prime}\left(z^{n}\right)$ acts as $V\left(z_{n}\right)$ on $\hat{U}_{n}^{\prime}\left(t^{n}\right) \Psi^{n}$ if both $\hat{U}_{n}^{\prime}\left(t^{n}\right) \Psi^{n}$ and its translate by $z_{n}$ is in the range of the $n$th projection $P_{n}^{Z}$. As the support of $\hat{U}_{n}^{\prime}\left(t^{n}\right) \Psi^{n}$ in $Z^{b_{n}}$-direction is restricted by the support of $\Phi_{\mathrm{Z}}^{n}$, we can find $J$ (taken $>L$ and $>Q$ ) such that for $n>J, \operatorname{Supp}\left(V\left(z_{L}\right) \Phi_{\mathrm{Z}}^{L}\right) \subset F_{n}^{b_{L}}$, consequently $\operatorname{Supp}\left(V\left(z_{n}\right) \Phi_{\mathrm{Z}}^{n}\right) \subset$ $F_{k_{n}}^{b_{n}}$. So, for $n>J$,

$$
(14)=\left\|\hat{U}\left(t_{n}\right) \Psi^{n}-\hat{U}_{n}^{\prime}\left(t^{n}\right) \Psi^{n}\right\|_{D_{n}}
$$

as $V\left(z_{n}\right)$ is unitary. For $n>J$ and $q=(k, u, v) \in D_{n}, \quad\left[\hat{U}\left(t_{n}\right)-\right.$ $\left.\hat{U}_{n}^{\prime}\left(t^{n}\right)\right] \Psi_{\mathrm{T}}^{n} \Lambda_{n}(q)=0$ for $k$ not in $F_{m_{n}}^{a_{n}}$, and otherwise

$$
\left[\hat{U}\left(t_{n}\right)-\hat{U}_{n}^{\prime}\left(t^{n}\right)\right] \Psi_{\mathrm{T}}^{n} \Lambda_{n}(q)=\Psi_{\mathrm{\top}}^{n} \Lambda_{n}(q)\left[\left\langle k, t^{n}\right\rangle-\left\langle k, t_{n}\right\rangle\right] .
$$

For the last expression to be non-zero, by Lemma 5.3, we must in particular have $k=\widehat{\kappa_{N}^{n}}\left(k_{N}\right)$ for some $k_{N} \in \mathrm{Z}^{a_{N}}$. As $\Psi_{\mathrm{T}}^{N}$ has finite support, the pointwise convergence of (13) in the proof of Proposition 6.2 can be made uniform on the $Z^{a_{N}}$-support of $\Psi_{\mathrm{T}}^{N}$, consequently also on the $Z^{a_{n}}$-support of $\Psi_{\mathrm{T}}^{n}$ (these two sets have the same number of elements). Thus, for $\epsilon>0$ there is an $R_{\epsilon}$ such that for $n>R_{\epsilon},\left|\left\langle k, t^{n}\right\rangle-\left\langle k, t_{n}\right\rangle\right|<\epsilon$ for all $k$ in the support of the $Z^{a_{n}}$-direction of $\Psi_{\mathrm{T}}^{n}$. Thus, by Fourier transforming back again, for $n>R_{\epsilon}$ (and $n>J)$,

$$
(14)<\epsilon \cdot\left\|\Psi^{n}\right\|_{D_{n}}=\epsilon \cdot\left\|\Phi^{n}\right\|_{E_{n}}=\epsilon \cdot\|\Phi\|_{G^{1}} .
$$

Convergence of the U's. The arguments are essentially as for translation, only the order of the steps is altered. Let $\gamma \in \widehat{G^{1}}$ as in Proposition 6.2. Again by Lemma 5.2, for $n>M,(U(\gamma) \Phi)^{n}=U^{n}\left(\gamma_{n}\right) \Phi^{n}$ where $\gamma_{n}=\left(u_{n}, g_{n}, s_{n}\right) \in \widehat{E_{n}}$. By preliminaries similar to those for translation, for $n>M$,

$$
\begin{gathered}
\left\|U(\gamma) \Phi-U_{n}^{\prime}\left(\gamma^{n}\right) \Phi\right\|_{G^{1}}=\| U\left(s_{n}\right) \Phi_{\mathrm{Z}}^{n} \hat{V}\left(u_{n}\right) \Psi_{\mathrm{\top}}^{n} \Lambda_{n} \\
-U_{n}^{\prime}\left(s^{n}\right) \Phi_{\mathrm{Z}}^{n} \hat{V}_{n}^{\prime}\left(u^{n}\right) \Psi_{\mathrm{T}}^{n} \Lambda_{n} \|_{D} n .
\end{gathered}
$$

With arguments as in the first part of the previous paragraph, there is $\hat{L}$ (taken $>M$ and $>Q$ ) such that $\hat{V}_{n}^{\prime}\left(u^{n}\right) \Psi_{\mathrm{\top}}^{n} \Lambda_{n}=\hat{V}\left(u_{n}\right) \Psi_{\mathrm{\top}}^{n} \Lambda_{n}$ for $n>\hat{L}$, and

$$
(15)=\left\|U\left(s_{n}\right) \Phi^{n}-U_{n}^{\prime}\left(s^{n}\right) \Phi^{n}\right\|_{E_{n}} .
$$


Let $\tilde{q}=(v, u, l) \in E_{n}$. Then, for $n>\hat{L}$, and $l \in F_{k_{n}}^{b_{n}}$ (otherwise the expression below in 0$),\left[U\left(s_{n}\right)-U_{n}^{\prime}\left(s^{n}\right)\right] \Phi^{n}(\tilde{q})=\left[\left\langle l, s_{n}\right\rangle-\left\langle l, s^{n}\right\rangle\right] \Phi^{n}(\tilde{q})$. Now, we follow the procedure of the last part of the proof for translation. Again, (15) will be bounded by $\epsilon \cdot\|\Phi\|_{G^{1}}$ for sufficiently large $n$.

6.2. Real numbers. The set up is as in Section 4. For $f \in C(\mathrm{R}), f_{a v}\left(k \epsilon_{n}\right)$, $k \in \mathbf{Z}_{n}$, is defined by $f_{a v}\left(k \epsilon_{n}\right)=\epsilon_{n}^{-1} \int_{(k-1 / 2) \epsilon_{n}}^{(k+1 / 2) \epsilon_{n}} f(x) d x, \epsilon_{n}=\sqrt{2 \pi / n}$.

Proposition 6.4. The group $\mathrm{R}$ is a limit, in the sense of Weyl systems, for the finite cyclic groups $\mathbf{Z}_{n}$, where $n$ runs through the odd positive integers.

Proof. Let $f \in C_{c}(\mathrm{R})$ be a continuous function with compact support inside $[-B, B], B>0$. We consider the isometries $R_{n}: l^{2}\left(\mathrm{Z}_{n}\right) \longrightarrow L^{2}(\mathrm{R})$ defined in Section 4.1 with associated projections $P_{n}$ as in Definition 2.1.

The fact that $P_{n} \longrightarrow$ id strongly is probably well-known. Nevertheless, we give a proof based on uniform convergence. If $\epsilon_{n}<1$, then $f_{n}:=P_{n} f=$ $\left.\sum_{|k| \leq n^{\circ}} f_{a v}\left(k \epsilon_{n}\right) \cdot\right|_{I_{n}^{k}}$ has support in $[-B-1, B+1]:=I(B)$. Furthermore, for any $x$ in this interval and $n^{\circ} \epsilon_{n}>B+1, x$ lies in some $I_{n}^{k}$, thus $f_{n}(x)=f_{a v}\left(k \epsilon_{n}\right)$. By the mean value theorem, $f_{a v}\left(k \epsilon_{n}\right)=f(z)$ for some $z$, also in $I_{n}^{k}$. So, for any $\epsilon>0$, just make $n$ large enough for $|f(y)-f(x)|<\epsilon$ for any pair $x, y \in I_{n}^{k}$, for all $k$ such that $I_{n}^{k} \cap I(B)$ is non-empty. Then $\left|f_{n}(x)-f(x)\right|<\epsilon$ for any $x$ in $I(B)$. Hence $f_{n} \longrightarrow f$ uniformly, which suffices for the $L^{2}$-convergence.

The result for $V_{n}^{\prime} \longrightarrow V$ follows very similarly.

As for $U_{n}^{\prime} \longrightarrow U$, it is easily seen that it is enough to check that $U_{n}^{\prime}\left(d^{n}\right) f_{n}$ gets close to the compression $(U(d) f)_{n}$. Here $d^{n} \in \mathbf{Z}_{n}$ approximates $d \in \mathbf{R}$ as in Section 4.1. So,

$$
\begin{aligned}
& \left|(U(d) f)_{n}-U_{n}^{\prime}\left(d^{n}\right) f_{n}\right| \\
& =\left|\sum_{|k| \leq n^{\circ}} \epsilon_{n}^{-1} \int_{(k-1 / 2) \epsilon_{n}}^{(k+1 / 2) \epsilon_{n}}\left(e^{i x d}-e^{\frac{2 \pi i}{n} k d^{n}}\right) f(x) d x \cdot\right|_{I_{n}^{k}} \mid \\
& \leq\left.\sum_{|k| \leq n^{\circ}}\|f\|_{\infty} \epsilon_{n}^{-1} \int_{(k-1 / 2) \epsilon_{n}}^{(k+1 / 2) \epsilon_{n}}\left|e^{i x d}-e^{i \epsilon_{n} k \epsilon_{n} d^{n}}\right| d x \cdot\right|_{I_{n}^{k} .}
\end{aligned}
$$

Since $(U(d) f)_{n}-U_{n}^{\prime}\left(d^{n}\right) f_{n}$ has support in $I(B)$ when $\epsilon_{n}<1$, consider the uniformly continuous function $G(x, y)=e^{i x y}$ on the compact strip $I(B) \times[d-1, d+1]$. So, given any $\epsilon>0$, we can find a uniform $n$ such that $\left|G(x, y)-G\left(x^{\prime}, y^{\prime}\right)\right|<\epsilon$ for any pair of points in each $I_{n}^{k} \times I_{n}^{y^{n}}$, for those $k \in \mathbf{Z}_{n}$ for which $I_{n}^{k} \cap I(B)$ is non-empty. Thus, for any $z \in I(B)$, there is a $k$ giving $\left|(U(d) f)_{n}(z)-\left(U_{n}^{\prime}\left(d^{n}\right) f_{n}\right)(z)\right|<\|f\|_{\infty}\left(\epsilon_{n}\right)^{-1} \int_{(k-1 / 2) \epsilon_{n}}^{(k+1 / 2) \epsilon_{n}} \epsilon d x=\|f\|_{\infty} \epsilon$. As this works uniformly, the theorem is correct. 


\section{Applications: $p$-adic Numbers and Rational Adeles}

Let $p$ be a prime number. Varadarajan mentions in [12] that the Weyl system on $Z_{p^{n}}$, as $n \rightarrow \infty$, converges to the Weyl system associated to the field of $\mathrm{p}$ adic numbers $\mathrm{Q}_{p}$. We construct examples of $H_{n}$ and $K_{n}$ in this case. These groups are interesting as they lead to phase spaces for $p$-adic quantum theories. Also, $\mathrm{Q}_{p}$ is the canonical example of a non-compact, non-discrete $G^{1}$ group.

7.1. The p-adic numbers. The field of p-adic numbers (see Gouvea [6] for basic properties) is the completion of the rational numbers $\mathrm{Q}$ under the $\mathrm{p}$ adic valuation $|\cdot|_{p}$. There is a (continuous) field structure on $\mathrm{Q}_{p}$ when $p$ is a prime. In a coordinate representation, the $p$-adic numbers can be viewed as Laurent series $x, x=\sum_{l=n}^{\infty} x_{l} p^{l}$, where $n$ is an integer and $x_{l} \in$ $\{0,1, \ldots, p-1\}$. Under the natural addition and multiplication, such that the resulting series also has coefficients in this set, $\mathrm{Q}_{p}$ is a field. Furthermore, under $|\cdot|_{p}$, defined by $|x|_{p}=p^{-n}$ where $x_{n}$ is the first non-zero coefficient in the series of $x$, these series are no longer just formal. In fact, $\mathrm{O}_{p}$ is a complete metric space. As an abelian topological group w.r.t. addition, $\mathrm{Q}_{p}$ is self-dual. The compact open subgroup $p^{n} O_{p}$ consists of those $x \in \mathrm{Q}_{p}$ for which $x_{k}=0$ for all $k$ smaller than the integer $n$. The p-adic integers $O_{p}$ form the maximal subring of $\mathrm{Q}_{p}$.

Put $H_{n}=p^{-a_{n}} O_{p}$ and $K_{n}=p^{b_{n}} O_{p}$, where $a_{n}+b_{n}=n$ and the integers $a_{n}, b_{n} \longrightarrow \infty$ as $n \longrightarrow \infty$. Then $H_{n} \nearrow \mathrm{Q}_{p}$ while $K_{n} \searrow\{0\}$, and through multiplication by $p^{a_{n}}, H_{n} / K_{n} \simeq \mathrm{Z}_{p^{n}}$. Thus, by Theorem $6.1, \mathrm{Q}_{p}$ is a limit of

$$
G_{n}=Z_{p^{n}} \quad(n \text { odd })
$$

in the sense of Weyl systems.

Notice that Schwartz-Bruhat functions on $\mathrm{O}_{p}$ are locally constant functions with compact support.

The measure on $Z_{p^{n}}$ from Paragraph 3.3.1, when $\mathrm{O}_{p}$ has its usual Haar measure and total measure of $O_{p}$ equals 1 , is the following: Then measure $\left(Z_{p^{n}}\right)=$ measure $\left(H_{n}\right)=p^{a_{n}}$, so $\operatorname{measure}(\{0\})=p^{a_{n}-n}=p^{-b_{n}}$. It makes sense from the approximation point of view that the measure of a point goes to zero, while the total measure goes to infinity. If $n$ is even and $a_{n}=n / 2$, then $\mathrm{Z}_{p^{n}}$ has the self-dual Haar measure of our set up.

7.2. Rational adeles. The locally compact abelian ring $A$ of adeles over $\mathrm{Q}$ (see Weil [17]) is defined as the product $\mathrm{R} \times A_{f}$, where $A_{f}$ is the group of finite adeles; the sequences $x=\left(x_{p}\right) \in \prod_{p \text { prime }} \mathrm{Q}_{p}$ such that $x_{p} \in O_{p}$ for all but finitely many places. These adeles define a locally compact ring under restricted product topology and pointwise addition and multiplication. Let 


$$
\begin{aligned}
& H_{n}=\mathrm{R} \oplus_{p \text { prime } \leq n} p^{-n} O_{p} \oplus_{p \text { prime }>n} O_{p} \quad \text { and } \\
& K_{n}=\{0\} \oplus_{p \text { prime } \leq n} p n O_{p} \oplus_{p \text { prime }>n} O_{p} .
\end{aligned}
$$

Then each $H_{n}$ is an open subgroup, and $H_{n} \nearrow \mathscr{A}$ while $K_{n} \subset H_{1}$ is compact, $K_{n} \searrow\{0\}$ and $H_{n} / K_{n} \simeq \mathrm{R} \oplus_{p \text { prime } \leq n} \mathrm{Z}_{p^{2 n}}$. Consequently, $\mathscr{A}$ is a limit of

$$
G_{n}=\mathbf{Z}_{n} \oplus_{p \text { prime } \leq n} \mathbf{Z}_{p^{2 n}} \quad(n \text { odd })
$$

in the sense of Weyl systems.

\section{REFERENCES}

1. F. Bruhat, Distributions sur un groupe localement compact et applications à l'étude des représentations des groupes p-adiques, Bull. Soc. Math. France 89 (1961), 43-75.

2. F. L. Chan, R. J. Finkelstein, q-deformation of the Coulomb problem, J. Math. Phys. 35 (1994), 3273-3284.

3. T. Digernes, V. S. Varadarajan, S. R. S. Varadhan, Finite approximations to quantum systems, Rev. Math. Phys. 6 (1994), 621-648.

4. E. Husstad, Endeligdimensjonale approksimasjoner til kvantesystemer, Diplomoppgave, University of Trondheim-NTH (1992) (in Norwegian).

5. R. J. Finkelstein, Transformation theory of q-quantum mechanics, Lett. Math. Phys. 34 (1995), 275-283.

6. F. Q. Gouvêa, p-adic Numbers, An Introduction, Springer (1993).

7. L. S. Pontrjagin, Topological Groups, second edition, Gordon and Breach (1966).

8. H. Reiter, Classical Harmonical Analysis and Locally Compact Groups, Oxford Univ. Press (1968).

9. J. Schwinger, Unitary operator bases, and The special canonical group, Proc. Nat. Acad. Sci. USA 46 (1960), 570-579, 1401-1405.

10. P. Štovíček, J. Tolar, Quantum mechanics in a discrete space-time, Rep. Math. Phys. 20 (1984), 157-170.

11. E. R. van Kampen, Locally bicompact abelian groups and their character groups, Ann. of Math. 36 (1935), 448-463.

12. V. S. Varadarajan, Variations on a theme of Schwinger and Weyl, Lett. Math. Phys. 34 (1995), 319-326.

13. V. S. Varadarajan, Path integrals for a class of p-adic Schrödinger equations, Lett. Math. Phys. 39 (1997), 97-106.

14. V. S. Vladimirov, On the spectrum of some pseudodifferential operators over the field of $p$ adic numbers, Leningrad Math. J. 2 (1991), 1261-1278.

15. V. S. Vladimirov, I. V. Volovich, I. Zelenov, Spectral theory in p-adic quantum mechanics, and representation theory, Soviet Math. Dokl. 41 (1990), 40-44, Math. USSR Izvestiya 36 (1991), 281-309.

16. A. Weil, Sur certains groupes d'opérateurs unitaires, Acta Math. 110 (1963), 143-211.

17. A. Weil, Basic Number Theory, Springer (1973).

18. H. Weyl, Theory of Groups and Quantum Mechanics, Dover (1931).

DIGERNES, HUSSTAD:

DEPARTMENT OF MATHEMATICAL SCIENCES

NORWEGIAN UNIVERSITY OF SCIENCE AND TECHNOLOGY

N-7034 TRONDHEIM, NORWAY

Trond.Digernes@math.ntnu.no

espen.husstad@storebrand.no
VARADARAJAN:

DEPARTMENT OF MATHEMATICS

UNIVERSITY OF CALIFORNIA

LOS ANGELES, CA 90095-1555, USA

vsv@math.ucla.edu 